Renate Schaub

\title{
Sponsoring und Doping
}

Beendigung von Sponsoringverträgen wegen Verfehlungen des Gesponserten, insbesondere in Dopingfällen 

Renate Schaub Sponsoring und Doping

This work is licensed under the Creative Commons License 2.0 "by-nd", allowing you to download, distribute and print the document in a few copies for private or educational use, given that the document stays unchanged and the creator is mentioned.

You are not allowed to sell copies of the free version.

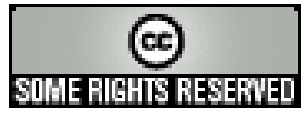


erschienen im Universitätsverlag Göttingen 2008 
Renate Schaub

Sponsoring und Doping

Beendigung von

Sponsoringverträgen wegen

Verfehlungen des Gesponserten, insbesondere in Dopingfällen

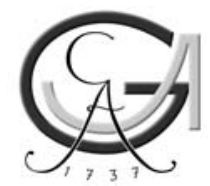

Universitätsverlag Göttingen 2008 


\section{Bibliographische Information der Deutschen Nationalbibliothek}

Die Deutsche Nationalbibliothek verzeichnet diese Publikation in der Deutschen Nationalbibliographie; detaillierte bibliographische Daten sind im Internet über $<$ http://dnb.ddb.de> abrufbar.

\section{Anschrift der Autorin}

Renate Schaub

E-Mail: ipr@ruhr-uni-bochum.de

Dieses Buch ist auch als freie Onlineversion über die Homepage des Verlags sowie über den OPAC der Niedersächsischen Staats- und Universitätsbibliothek (http://www.sub.uni-goettingen.de) erreichbar und darf gelesen, heruntergeladen sowie als Privatkopie ausgedruckt werden. Es gelten die Lizenzbestimmungen der Onlineversion. Es ist nicht gestattet, Kopien oder gedruckte Fassungen der freien Onlineversion zu veräußern.

Satz und Layout: Renate Schaub

Umschlaggestaltung: Margo Bargheer

(C) 2008 Universitätsverlag Göttingen

http://univerlag.uni-goettingen.de

ISBN: 978-3-940344-54-0 


\section{Inhaltsverzeichnis}

I. Einleitung .................................................................................................. 7

II. Grundlagen der rechtlichen Beurteilung von Sponsoringverträgen .......... 9

1. Begriffsbestimmung................................................................................... 9

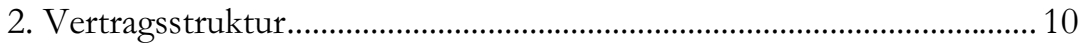

3. Rechtliche Beurteilung ......................................................................... 11

III. Beendigungsmöglichkeiten aufgrund eines vertraglich vereinbarten Kündigungsrechts ................................................................................... 13

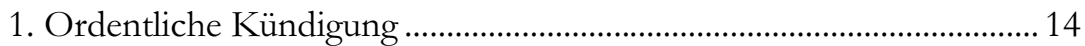

2. Außerordentliche Kündigung .............................................................. 14

a) Voraussetzungen............................................................................ 14

(1) Verstoß gegen wesentliche vertragliche

Verpflichtungen oder gesetzliche Vorschriften.............. 15

(2) Leistungsstörungen auf Seiten des Gesponserten .......... 18

b) Rechtsfolgen ........................................................................................ 19

IV. Beendigung aufgrund gesetzlicher Regelungen........................................ 23

1. Verfehlung des Gesponserten als Leistungsstörung ............................. 23

2. Verfehlung des Gesponserten als wichtiger Grund für eine außerordentliche Kündigung des Vertrags ............................................ 25

a) Kündigung durch den Sponsor nach \ 649 BGB .............................. 26

b) Kündigung durch den Sponsor nach \ 314 BGB ............................. 27

(1) Verhältnis zu anderen Kündigungsregelungen...............27

(2) Voraussetzungen einer Kündigung nach \ 314 BGB ... 28

(3) Rechtsfolgen einer Kündigung nach \314 BGB........... 32

3. Abgrenzung zu Störung bzw. Wegfall der Geschäftsgrundlage, 
V. Alternative vertragsrechtliche Möglichkeiten

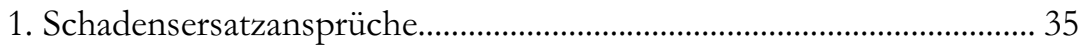

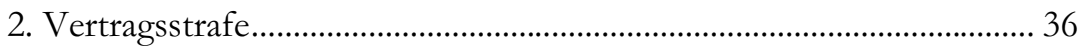

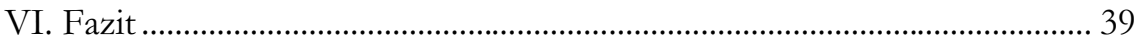

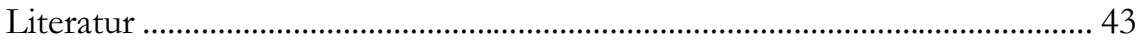




\section{Einleitung}

Die zunehmende Aufdeckung von Dopingfällen beim Radsport, aber auch bei anderen Sportarten, hat zu einer Verstärkung der Diskussion über juristische Aspekte des Dopings geführt. Bisher standen dabei strafrechtliche ${ }^{1}$ sowie vereins- bzw. verbandsrechtliche ${ }^{2}$ Fragen im Mittelpunkt; sofern die schuldrechtlichen Konsequenzen des Dopings erörtert wurden, ging es überwiegend um Ansprüche des Gedopten, von Vereinen oder Veranstaltern, von Zuschauern und vereinzelt auch von Sponsoren ${ }^{3}$. Hier soll eine

${ }^{1}$ Siehe insb. Kühl, in: Vieweg (Hrsg.), Doping. Realität und Recht, S. 77 ff.; Cherkeh, Betrug ( 263 StGB), verübt durch Doping, S. 63 ff.; Fritzweiler/ Pfister/ Summerer-Reinhart, Praxishandbuch Sportrecht, 8. Teil Rn. 138 ff.; Schild, in: Schild (Hrsg.), Rechtliche Fragen des Dopings, S. 13 ff.; Otto, SpuRt 1994, 10 ff.; Fritzweiler, SpuRt 1998, 234 f.; Cherkeh/ Momsen, NJW 2001, 1745 ff.; Heger, SpuRt 2001, 92 ff.; dens., JA 2003, 76 ff.; dens., SpuRt 2007, 153 ff.; Grotz, SpuRt 2005, 93 ff.; Glauben, DRiZ 2006, 308 f.; Leipold, NJW-Spezial 06, 423 f.; Bannenberg, SpuRt 2007, 155 f.; Hauptmann/ Rübenstabl, MedR 2007, 271 ff.; Bruggmann/ Grau, PharmR 2008, 101 ff.; weitere, umfassende Nachweise bei MünchKommStGB-Freund, \6a AMG, zu Beginn.

2 Siehe insb. Steinigen, Zivilrechtliche Aspekte des Dopings aus der Sicht des Spitzensportlers; Vieweg, NJW 1991, 1511 ff.; dens., in: Vieweg (Hrsg.), Doping. Realität und Recht, S. 113 ff.; Adolphsen, SpuRt 2000, 97 ff.; Menzel, WRP 2002, 810 ff.; Horst/ Jacobs, RdA 2003, 215 ff.; Mertens, SpuRt 2006, 177 ff.; Petri, SpuRt 2003, 230 ff.; dens., SpuRt 2006, 105 ff.

${ }^{3}$ Siehe nur Schwab, in: Schild (Hrsg.), Rechtliche Fragen des Dopings, S. 35 ff.; Gutheil, Doping. Die haftungsrechtliche und sportrechtliche Verantwortung von Sportler, Trainer, Arzt und Sportverband, S. 106 ff., wo Ansprüche von Sponsoren allerdings nicht angesprochen werden; Walker, in: Vieweg (Hrsg.), Doping. Realität und Recht, S. 135, 151 ff. (arbeitsrechtliche Probleme); Bergermann, Doping und Zivilrecht. Rechtmäßigkeit von Doping-Sanktionen sowie durch Doping begründete Schadensersatzansprüche, S. 153 ff. (mit kurzen Ausführungen zur Vertragsauflösung S. 238 ff.); Steinigen, Zivilrechtliche Aspekte des Dopings aus der Sicht des Spitzensportlers (ohne Einbeziehung der Ansprüche von Sponsoren); Turner, NJW 1992, 720 ff.; dens., Festgabe Zivilrechtslehrer 1934/35, 669 ff.; Friedrich, SpuRt 1995, 8 ff.; Holzer/Fritzweiler, in: Fritzweiler (Hrsg.), Doping. Sanktionen Beweise Ansprüche, S. 57 ff. - Eine neuere Abhandlung befasst sich mit Vertragsstrafen in 
Problematik in den Vordergrund gestellt werden, deren praktische Relevanz die zahlreichen Berichte über Rückzüge von Sponsoren der Tour de France verdeutlichen: die Frage, ob und unter welchen Voraussetzungen Sponsoringverträge in Fällen von Verfehlungen des Gesponserten, also auch bei Doping, beendet werden können. In Pressemitteilungen zu Dopingfällen ist häufig davon die Rede, dass Sponsoren ihre Verträge mit gedopten Sportlern beenden würden, weil dort entsprechende Rücktrittsklauseln vorgesehen seien. Daher soll zum einen untersucht werden, wie derartige Rücktrittsklauseln beschaffen sein können und sollten, um rechtlicher Überprüfung standzuhalten. Zum anderen soll der Frage nachgegangen werden, unter welchen Voraussetzungen auch ohne ausdrückliche Regelung in Dopingfällen eine Vertragsbeendigung nach allgemeinen schuldrechtlichen Grundsätzen in Betracht kommt. Auch die Rechtsfolgen einer Vertragsbeendigung sollen dargestellt werden, insbesondere ihr Verhältnis zu anderen vertragsrechtlichen Folgen des Dopings, wie vor allem Schadensersatzansprüchen oder Vertragsstrafen. In der Regel wird es sich bei Sportsponsoringverträgen um Dauerschuldverhältnisse handeln, so dass die Untersuchung auf Voraussetzungen und Rechtsfolgen einer Kündigung des Sponsoringvertrags begrenzt wird.

Die Voraussetzungen und Rechtsfolgen der Vertragsbeendigung hängen von der rechtlichen Einordnung des Sponsoringvertrags ab. Daher sind zunächst Vertragsstruktur und wichtigste Pflichten der Vertragspartner als Grundlagen der vertragsrechtlichen Beurteilung darzustellen (II.). Danach sollen die Kündigungsmöglichkeiten aufgrund eines vertraglich vereinbarten Kündigungsrechts unter Berücksichtigung der in Formularverträgen üblicherweise vorgeschlagenen Klauseln (III.) sowie aufgrund gesetzlicher Regelungen (IV.) erörtert werden. Die Vertragsbeendigung aufgrund gesetzlicher Regelungen dürfte vor allem für «Kleinsponsoren» von Bedeutung sein, deren Verträge keine speziellen Beendigungsregelungen enthalten. Sie bildet jedoch zugleich die Grundlage für Auslegung und ggf. AGB-Kontrolle von Kündigungsklauseln, wie sie sich in umfangreicheren Sponsoringvertragswerken regelmäßig finden. Nach einer kurzen Darstellung alternativer vertragsrechtlicher Möglichkeiten (V.) werden die Voraussetzungen der Vertragsbeendigung sowie die Möglichkeiten der Vertragsgestaltung zusammengefasst (VI.).

Sponsoringverträgen (Nesemann, NJW 2007, 2083 ff.), zwei weitere mit Kündigungsmöglichkeiten kraft Gesetzes (Kratz/ Quantius, Festschrift Fenn, 2000, 177 ff.; Humberg, JR 2005, 271 ff.). 


\section{Grundlagen der rechtlichen Beurteilung von Sponsoringverträgen}

\section{Begriffsbestimmung}

Die Bezeichnung «Sponsoringvertrag» wird für eine Reihe unterschiedlicher Vertragstypen verwendet; der Sprachgebrauch ist uneinheitlich ${ }^{4}$. Hier soll unter Sponsoring ein Rechtsgeschäft verstanden werden, bei dem der Gesponserte eine Kommunikationsleistung (durch aktive Werbung oder durch das Verschaffen von Möglichkeiten zur werblichen Nutzung von Rechtspositionen, die durch die Tätigkeit oder Persönlichkeit des Gesponserten geprägt sind) erbringt und dafür vom Sponsor eine Förderleistung in Form einer Geldzahlung, Sachzuwendung, Gebrauchsüberlassung, Werkoder Dienstleistung (oder eine Kombination solcher Leistungen) erhält. Zugleich wird im Rahmen dieses Rechtsgeschäfts eine überindividuellen Zwecken dienende Aktivität des Gesponserten (Sponsoringobjekt) gefördert, deren Durchführung auch zur zusätzlichen Vertragspflicht des Ge-

${ }^{4}$ Mitunter wird - in Anknüpfung an betriebswirtschaftliche Ansätze - die Funktion des Sponsorings als Instrument der Unternehmenskommunikation in den Vordergrund gestellt (siehe aus der juristischen Literatur insb. Röhrborn, Der Sponsoringvertrag als Innengesellschaft, S. 35; Creifelds, Rechtswörterbuch [hrsg. von Weber], Stichwort «Sponsoring»), teilweise wird der Sponsoringbegriff auf einzelne Bereiche beschränkt, z.B. auf das Medien- bzw. Sendungssponsoring ( $\int 2$ II Nr. 7 Rundfunkstaatsvertrag, z.B. Deutsches Rechtslexikon [hrsg. von Tilch/ Arnold], Stichwort «Sponsoring») oder auf das Veranstaltungssponsoring (Köbler, Juristisches Wörterbuch, Stichwort «Sponsoring»). Umfassendere Sponsoringdefinitionen finden sich insb. bei Brubn/ Mehlinger, Rechtliche Gestaltung des Sponsoring, Bd. I, S. 4; Otten, Sponsoring: Erscheinungsformen, Rechtsgrundlagen und Bedeutung für die Finanzierung des öffentlich-rechtlichen Rundfunks, S. 15; Kolvenbach, AnwBl 1998, 289, 291; Weiand, Kultur- und Sportsponsoring im deutschen Recht, unter besonderer Berücksichtigung urheber-, medien- und wettbewerbsrechtlicher Aspekte, S. 30; dems., NJW 1994, 227, 230. 
sponserten erhoben werden kann 5 . Innerhalb dieser Definition lassen sich je nach dem von den Vertragspartnern angestrebten Verhältnis von Leistung und Gegenleistung ${ }^{6}$ verschiedene Konstellationen von Sponsoringverträgen unterscheiden ${ }^{7}$ : Die beim Sportsponsoring häufigste Variante ist diejenige, bei der die Vertragspartner von einer Gleichwertigkeit von Leistung(en) und Gegenleistung(en) ausgehen; nur solche voll entgeltlichen Verträge sind Gegenstand der folgenden Erörterungen. Der Vollständigkeit halber sei erwähnt, dass es auch Sponsoringverträge gibt, bei denen der Wert der Leistung(en) des Gesponserten nach den Vorstellungen der Beteiligten niedriger ist als der Wert der Sponsorenleistungen(en); dann liegt ein teilweise entgeltlicher Vertrag vor; dieser Typus findet sich häufig beim Sozio- oder Umweltsponsoring 8 .

\section{Vertragsstruktur}

Beim Sponsoring des hier untersuchten Typus erbringt der Sponsor eine oder mehrere Förderleistungen, beispielsweise in Form von (einmaligen oder wiederkehrenden) Geldleistungen, Sachleistungen (z.B. Überlassung von Ausrüstungsgegenständen, Bereitstellung von Infrastruktur) oder durch bestimmte Tätigkeiten (z.B. Beratung des Gesponserten, Service für Ausrüstungsgegenstände, Organisationstätigkeiten). Auf Seiten des Gesponserten steht dem eine «Kommunikationsleistung» gegenüber: der Gesponserte wirbt entweder aktiv für den Sponsor (z.B. durch Sponsorenhinweise) oder stellt ihm Nutzungsmöglichkeiten zur Verfügung (z.B. indem er ihm Werbemöglichkeiten bereitstellt oder ihm Rechtspositionen verschafft, die durch das Persönlichkeitsrecht des Gesponserten geprägt sind, wie etwa die Befugnis zur Verwendung von Namen oder Bild des Gesponserten). Diese Kommunikationsleistung des Gesponserten ist prägend für den sich allmählich herausbildenden Typus des Sponsoringver-

${ }^{5} \mathrm{Zu}$ Entwicklung und Implikationen dieser Sponsoringdefinition siehe Schaub, Sponsoring und andere Verträge zur Förderung überindividueller Zwecke, S. 18, 390, $476 \mathrm{ff} ., 723 \mathrm{f}$.

${ }^{6}$ Maßgeblich muss hier die Beurteilung der Entgeltlichkeit bzw. Teil-Entgeltlichkeit durch die Vertragspartner sein (Prinzip der subjektiven Äquivalenz, siehe dazu insb. Fischer, Die Unentgeltlichkeit im Zivilrecht, S. 53 ff. m.w.N.).

${ }^{7}$ Umfassend zu den unterschiedlichen Konstellationen von Sponsoringverträgen Schaub, Sponsoring und andere Verträge zur Förderung überindividueller Zwecke, S. 390 ff., 622 ff.

${ }^{8}$ Dieser Vertragstypus lässt sich wiederum in zwei Untervarianten unterteilen, je nachdem, ob die Verwirklichung des Sponsoringobjekts nach dem Willen der Beteiligten als zusätzliche Pflicht des Gesponserten anzusehen ist oder nicht. 
trags, denn sie stellt im Unterschied zu den unterschiedlichen Ausprägungen der Sponsorenleistungen die vertragstypische Leistung dar. Auch wenn der Vertragsname die Leistung des Sponsors in den Vordergrund stellt, ist daher die Kommunikationsleistung des Gesponserten der Ausgangspunkt der rechtlichen Beurteilung des Sponsoringvertrags.

\section{Rechtliche Beurteilung}

Der Sponsoringvertrag als atypischer Vertrag lässt sich mit Hilfe schuldrechtlicher Regelungen nur partiell erfassen. Berücksichtigt man, dass sich dieser Vertragstypus neben den gesetzlich geregelten Vertragstypen und zu deren Ergänzung in der Praxis herausgebildet hat, erscheint es sinnvoll, bei jedem einzelnen Sponsoringvertrag zunächst anhand der konkreten Parteivereinbarung Regelungszwecke und -ziele zu ermitteln und danach gegebenenfalls ergänzend dispositives Gesetzesrecht zur Verwirklichung dieser Ziele heranzuziehen'. Sofern die Parteivereinbarung im Sponsoringvertrag nicht erschöpfend ist und sich die Lücken nicht ohne weiteres durch ergänzende Vertragsauslegung schließen lassen, muss auf die Vorschriften über gesetzlich geregelte Vertragstypen zurückgegriffen werden, mit deren Hilfe sich die Leistungen der Beteiligten beim Sponsoringvertrag am besten erfassen lassen ${ }^{10}$.

Für die Fälle einer ergänzenden Heranziehung vertragsrechtlicher Regelungen ist zwischen den unterschiedlichen Ausprägungen der vertragstypischen Kommunikationsleistung des Gesponserten zu differenzieren: Wirbt der Gesponserte aktiv für den Sponsor, erbringt er i.d.R. eine Werkleistung i.S.d. $\iint 631 \mathrm{ff} . \mathrm{BGB}^{11}$. Verschafft er dem Sponsor hingegen Nutzungsmöglichkeiten, z.B. indem er Werbemöglichkeiten bereitstellt, dürften i.d.R. die Vorschriften über die Rechtspacht ( $\iint 581$ ff. BGB) anwendbar sein, und beim Verschaffen persönlichkeitsgeprägter Rechtspositionen kommt - je nach Umfang der verschafften Rechtspositionen - eine Anwendung der Regeln über Rechtskauf ( $\$ \int 453,433$ ff. BGB) oder Rechts-

${ }^{9} \mathrm{Zu}$ diesem in erster Linie vertragsorientierten Ansatz (im Gegensatz zum in erster Linie gesetzesorientierten Ansatz) bei der Beurteilung atypischer Verträge siehe hier nur Lieb, DB 1988, 946; Oechsler, Gerechtigkeit im modernen Austauschvertrag, S. 275, 280 ff.; Stoffels, Gesetzlich nicht geregelte Schuldverträge, insb. S. 31 f., 115 ff., 160 ff., 175 ff., 192, auch mit Nachweisen zur Gegenansicht (S. 147 f.).

${ }^{10} \mathrm{Zu}$ Einzelheiten der methodischen Vorgehensweise siehe Schaub, Sponsoring und andere Verträge zur Förderung überindividueller Zwecke, S. 63 ff.

${ }^{11}$ Die - selteneren - Fälle, in denen die Werbung für den Sponsor als Dienstleistung

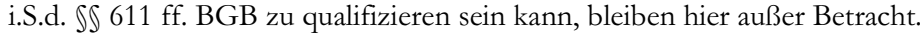


pacht ( $\iint 581$ ff. BGB) in Betracht. In Bezug auf die Sponsorenleistung können - je nach Art der Leistung - Elemente von Kauf-, Miet- oder Geschäftsbesorgungsvertrag eine Rolle spielen. Führt die ergänzende Anwendung derartiger Regelungen zu einer Normenhäufung, sollten die Regeln der unterschiedlichen Vertragstypen miteinander kombiniert werden, solange dies nicht zu Wertungswidersprüchen führt; bei Wertungswidersprüchen sollten diejenigen Vorschriften angewandt werden, die für den dominierenden Vertragsteil, also hier die Leistung des Gesponserten als vertragstypische Leistung, gelten. Bei Normenmangel kommt hingegen in erster Linie eine ergänzende Heranziehung von Vorschriften des allgemeinen Schuldrechts in Betracht. Auf der Grundlage dieser methodischen Prämissen ist zunächst die Beendigung von Sponsoringverträgen aufgrund eines vertraglich vereinbarten Kündigungsrechts zu analysieren, anschlieBend die Beendigung aufgrund gesetzlicher Kündigungsregelungen. 


\section{Beendigungsmöglichkeiten aufgrund ei- nes vertraglich vereinbarten Kündigungsrechts}

Sponsoringverträge enthalten häufig Klauseln über Voraussetzungen und Rechtsfolgen einer Vertragsbeendigung ${ }^{12}$. Bei Verfehlungen des Gesponserten, wie z.B. Doping ${ }^{13}$, kann der Sponsor entweder vertraglich festgelegte Möglichkeiten der ordentlichen Kündigung nutzen oder sich auf ein Recht zur außerordentlichen Kündigung berufen. Die Formularbücher gehen regelmäßig von langfristig angelegten Sponsoringverträgen aus ${ }^{14}$ und enthalten Vorschläge für ein ordentliches und ein außerordentliches Kündigungsrecht ${ }^{15}$. Denkbar erscheint es auch, im Rahmen der Privatautono-

12 Siehe insb. Beck'sches Formularbuch-Feick, Sponsoringvertrag (S. 1276 ff.), \S 7, 8; Münchener Vertragshandbuch-Weiand, Bd. 3, Wirtschaftsrecht II, Sportsponsoringvertrag (S. 1237 ff.), \8; Weiand/ Poser, Sponsoringvertrag, \ 9; Partikel, Formularbuch für Sportverträge, D. III. 2. Sport-Sponsoringvertrag (S. 282 ff.), \ 4, D. III. 3. (Ausführlicher) Sport-Sponsoringvertrag (S. 286 ff.), \ 10, keine derartige Regelung ist hingegen im Formular über einen Sportereignis-Sponsoring-Vertrag zu finden (D. III. 7 [S. 304 ff.]).

${ }^{13}$ Die Definition von Doping ist nicht einheitlich (siehe dazu nur MünchKommStGBFreund, S 6a AMG Rn. 30; Bruggmann/ Grau, PharmR 2008, 101; Deutsch, VersR 2008, 145, 146 f. m.w.N.); sie ist jedoch für die hier untersuchten Fragen nicht entscheidend, insbesondere kommt es nicht ausschließlich auf die strafrechtliche Definition nach dem AMG an, sondern darauf, was nach dem Vertrag als Verfehlung des Gesponserten anzusehen ist. Eine solche Verfehlung kann auch bereits unterhalb der Strafbarkeitsschwelle vorliegen.

14 Ausnahme: Partikel, Formularbuch für Sportverträge, D. III. 7. SportereignisSponsoring-Vertrag (S. 304 ff.).

15 Beck'sches Formularbuch-Feick, Sponsoringvertrag (S. 1276 ff.), \S 7, 8 (nur außerordentliches Kündigungsrecht); Münchener Vertragshandbuch-Weiand, Bd. 3, Wirtschaftsrecht II, Sportsponsoringvertrag (S. 1237 ff.), \ 8; Weiand/ Poser, Sponsoringvertrag, \ 9; Partikel, Formularbuch für Sportverträge, D. III. 2. SportSponsoringvertrag (S. 282 ff.), \4; abweichend in Bezug auf die ordentliche Kündigung (feste Vertragslaufzeit mit Erstverhandlungsoption für den Sponsor für die weitere Spielsaison) D. III. 3. (Ausführlicher) Sport-Sponsoringvertrag (S. 286 ff.), $\int 10$. 
mie in Sponsoringverträge mit einmaligem Leistungsaustausch eigenständige Rücktrittsregeln aufzunehmen. Die Voraussetzungen und Rechtsfolgen eines solchen Rücktrittsrechts könnten ähnlich ausgestaltet werden wie diejenigen einer außerordentlichen Kündigung.

\section{Ordentliche Kündigung}

Kann der Sponsor eine im Vertrag vorgesehene Möglichkeit der ordentlichen Kündigung nutzen, ergeben sich keine besonderen rechtlichen Probleme, da diese Art der Kündigung regelmäßig nur die Einhaltung der vereinbarten Kündigungsfrist voraussetzt, es also auf Vorliegen oder gar Schwere einer Verfehlung des Gesponserten nicht ankommt. Die ordentliche Kündigung wirkt ex nunc, so dass die erbrachten Leistungen nicht zurück zu gewähren sind ${ }^{16}$.

Zu prüfen ist in Dopingfällen vor allem, ob bzw. wie stark es dem Image des Sponsors im konkreten Fall abträglich ist, wenn er sein Sponsoringengagement noch bis zum Kündigungstermin fortsetzt. Basiert beispielsweise die Werbestrategie des Sponsors auf Reinheit oder Unverfälschtheit, kann schon die befristete Fortsetzung des Sponsoringengagements in einem Dopingfall zu nicht mehr zumutbaren Imageverlusten führen. Daher dürfte es hier, wie auch in den meisten Fällen anderer Verfehlungen des Gesponserten, entscheidend auf die Möglichkeit einer auBerordentlichen Kündigung ankommen.

\section{Außerordentliche Kündigung}

\section{a) Voraussetzungen}

In den Formularen für Sponsoringverträge wird meist festgelegt, dass beide Vertragsparteien berechtigt sind, den Vertrag aus wichtigem Grund fristlos zu kündigen. Der wichtige Grund sollte anschließend vertragsspezifisch konkretisiert werden. Mitunter findet sich die Formulierung, ein wichtiger Grund liege insbesondere vor, wenn die andere Vertragspartei schuldhaft gegen ihr obliegende wesentliche vertragliche Verpflichtungen oder gegen

\footnotetext{
16 Siehe insb. Münchener Vertragshandbuch-Weiand, Bd. 3, Wirtschaftsrecht II, Sportsponsoringvertrag (S. 1237 ff.), \ 8 I; Weiand/Poser, Sponsoringvertrag, \ 9 I.
} 
gesetzliche Vorschriften verstoßen habe ${ }^{17}$. Bei Verletzung gesetzlicher Vorschriften soll bereits der qualifizierte Verdacht eines Verstoßes gegen eine solche Regelung ausreichen ${ }^{18}$. Weiterhin wird die Vereinbarung eines Kündigungsrechts des Sponsors vorgeschlagen, das insbesondere dann eingreifen soll, wenn der Gesponserte die gesponserten Aktivitäten nicht weiter entfaltet oder seine Kommunikationsleistung nicht erbringt bzw. nicht erbringen kann ${ }^{19}$.

\section{(1) Verstoß gegen wesentliche vertragliche Verpflichtungen oder gesetzliche Vor- schriften}

War der Gesponserte gedopt, dürfte regelmäßig ein Verstoß gegen gesetzliche Vorschriften vorliegen. Der Gesponserte kann sich strafbar gemacht haben: beim unerlaubten Besitz von Substanzen, die in den Anlagen zum BtMG aufgeführt sind, nach $\iint 29,29 \mathrm{a} \mathrm{BtMG}{ }^{20}$, eventuell auch nach $\ 263$ StGB im Verhältnis zum Sponsor ${ }^{21}$, weiterhin nach $\int 95$ I Nr. 2b i.V.m. \6a IIa AMG bei Besitz von Arzneimitteln in nicht geringer Menge zu Dopingzwecken im Sport ${ }^{22}$. Sinnvoll erscheint es, auch Regelverstöße

${ }^{17}$ Münchener Vertragshandbuch-Weiand, Bd. 3, Wirtschaftsrecht II, Sponsoringvertrag (S. 1237 ff.), \ 8 II; Weiand/ Poser, Sponsoringvertrag, \9 II.

18 Münchener Vertragshandbuch-Weiand, Bd. 3, Wirtschaftsrecht II, Sportsponsoringvertrag (S. 1237 ff.), \ 8 II lit. b S. 2; Weiand/ Poser, Sponsoringvertrag, \ 9 II lit. b S. 2.

${ }^{19}$ Münchener Vertragshandbuch-Weiand, Bd. 3, Wirtschaftsrecht II, Sportsponsoringvertrag (S. 1237 ff.), \ 8 III; Weiand/Poser, Sponsoringvertrag, \$ 9 II lit. c, III.

${ }^{20}$ Dazu z.B. Otto, SpuRt 1994, 10, 15; Glauben, DRiZ 2006, 308 f. Diese Vorschriften umfassen jedoch nur wenige Dopingmittel, siehe insb. Hauptmann/ Rübenstabl, MedR 2007, 271, 274; Bruggmann/ Grau, PharmR 2008, 101, 103.

21 Die Strafbarkeit nach $\int 263$ StGB ist allerdings in der strafrechtlichen Literatur umstritten, siehe dazu z.B. Cherkeh, Betrug (\$ 263 StGB), verübt durch Doping, S. 78 ff.; Fritzweiler/ Pfister/ Summerer-Reinhart, Sportrecht, 8. Teil Rn. 138 ff.; Cherkeh/ Momsen, NJW 2001, 1745, 1748 ff.; Grotz, SpuRt 2005, 93, 94 ff.; Leipold, NJW-Spezial 2006, 423; Glauben, DRiZ 2006, 308, 309; Kargl, NStZ 2007, 489, 493; Bruggmann/ Grau, PharmR 2008, 101, 102 f.

22 Teilweise ist umstritten, ob die Neufassung der Regelung durch das Gesetz zur Verbesserung der Bekämpfung des Dopings im Sport tatsächlich zu einer Strafbarkeit des Sportlers führt, insbesondere, ob dies dem Schutzzweck des gesamten Regelungskomplexes entspricht (dazu z.B. Hauptmann/ Rübenstabl, MedR 2007, 271, 276 f.; Kargl, NStZ 2007, 489, 490 ff.; Bruggmann/ Grau, PharmR 2008, 101, 105 f.; Deutsch, VersR 2008, 145, 146, 148). Der Wortlaut des \6a IIa AMG spricht jedoch für eine Strafbarkeit, weil nur vom Besitzen zu Dopingzwecken die Rede ist, nicht - wie etwa in Abs. I - vom Doping bei anderen. Zudem sprechen auch die internationalen Verpflichtungen der Bundesrepublik Deutschland (vor allem Art. 8 Abs. II 
unterhalb der Strafbarkeitsschwelle als Gründe für die außerordentliche Kündigung zu normieren, etwa Zuwiderhandlungen gegen die Regelungen einzelner Sportverbände ${ }^{23}$, gegen die Regelwerke des Deutschen Sportbunds $^{24}$, der Nationalen Anti-Doping-Agentur Deutschland (NADA) ${ }^{25}$ oder der World Anti-Doping Agency (WADA) ${ }^{26}$. Empfehlenswert ist die Aufnahme einer ausdrücklichen Verweisung auf die für den jeweiligen Gesponserten maßgeblichen Regelwerke in die Klauseln über die außerordentliche Kündigung.

Will man - wie in gängigen Sponsoringvertragsformularen vorgesehen - bereits den qualifizierten Verdacht eines Verstoßes für die außerordentliche Kündigung ausreichen lassen, muss für die Konkretisierung, wann ein solcher qualifizierter Verdacht vorliegt, ein angemessener Ausgleich zwischen den Interessen der Beteiligten gefunden werden: Auf Seiten des Sponsors ist insbesondere der beim Sponsoringvertrag regelmäßig bezweckte Imagetransfer vom Gesponserten zum Sponsor zu berücksichtigen. Dieser wird bereits beim Dopingverdacht geschmälert, sodass es gerechtfertigt erscheint, die Schwelle für einen Verstoß niedriger anzusetzen als bei anderen Vertragstypen. Andererseits sind auch Fälle bekannt, in denen die Bekanntheit des Sponsors gerade durch Dopingskandale zunahm und dadurch Absatzsteigerungen zu verzeichnen waren ${ }^{27}$. Dies dürf-

des Internationalen Übereinkommens gegen Doping im Sport [BR-Drs. 709/06, S. 14], wonach die Vertragsstaaten Maßnahmen ergreifen, um «die Anwendung und den Besitz verbotener Wirkstoffe und Methoden durch Athleten im Sport zu verhüten und einzuschränken, es sei denn, die Anwendung erfolgt aufgrund einer Ausnahmegenehmigung zur therapeutischen Anwendung») dafür, den Sportler selbst in den Straftatbestand einzubeziehen. Ob die Strafbarkeit des Sportlers nach \ 95 I Nr. 2b i.V.m. \6a IIa AMG allerdings große praktische Bedeutung erlangen wird, bleibt abzuwarten.

23 Auf die Bestimmungen der einzelnen Fachverbände, die häufig an die Rahmenrichtlinien des DSB angelehnt sind, wird hier nicht näher eingegangen.

24 Rahmenrichtlinien des DSB zur Bekämpfung des Dopings, letzte Änderung 1.12.2001, abrufbar unter http://www.leichtathletik.de/dokumente/ergebnisse/uploads/antidoping/handbuch/RahmenrichtlinienDSB_1.pdf.

25 Anti-Doping-Regelwerk der Nationalen Anti-Doping-Agentur (NADA-Code), Stand: 1.1.2006, abrufbar unter http://www.nada-bonn.de/fileadmin/user_upload/ Downloads/Regelwerke/NADA-Code_2006.pdf.

26 World Anti-Doping Code (2003), abrufbar unter http:/ / www.wadaama.org/rtecontent/document/code_v3_pdf; Neufassung 2009 abrufbar unter http://www.wada-ama.org/rtecontent/document/code_v2009_En.pdf.

27 Z.B. für Festina nach dem Ausschluss von der Tour de France 1998, vgl. dazu z.B. Kratz/ Quantius, Festschrift Fenn, 177, 187; Bergermann, Doping und Zivilrecht, S. 242. - Bergermann, a.a.O., S. 240, geht davon aus, dass sogar in derartigen Fällen eine Kündigung aus wichtigem Grund in Betracht komme, denn es könne dem 
ten jedoch seltene Ausnahmekonstellationen sein, denen gegebenenfalls durch eine einzelfallbezogene Vertragsauslegung Rechnung getragen werden kann. Auf Seiten des Gesponserten ist zu berücksichtigen, dass jedenfalls in Bezug auf Straftatbestände zunächst die Unschuldsvermutung gilt und dass unberechtigte Anschuldigungen in den Medien in ihrer negativen Wirkung auf Person und Tätigkeit durch eine vorzeitige Beendigung des Sponsoringvertrags - als am weitesten reichende vertragsrechtliche Sanktion - möglicherweise über Gebühr verstärkt werden können.

Diese differenzierte Interessenlage zeigt, dass generalisierende Lösungen in diesem Punkt kaum zu finden sein dürften. Will man bereits den qualifizierten Verdacht eines Verstoßes gegen gesetzliche Regelungen als Kündigungsgrund ausreichen lassen, empfiehlt sich daher dringend eine vertragsspezifische Konkretisierung, bei der insbesondere die Werbestrategie des Sponsors zu berücksichtigen ist. Ausgangsfrage sollte sein, ob der Imagetransfer als wesentliches Vertragsziel bereits beim Verdacht eines Verstoßes in Frage steht ${ }^{28}$. Da die Folgen für den Sponsor im Einzelfall meist nicht sicher prognostiziert werden können, empfiehlt sich eine vorherige Festlegung durch die Parteien, ob dem Image des Sponsors bereits der qualifizierte Verdacht eines Verstoßes des Gesponserten abträglich ist oder ob angesichts der gesamten Werbestrategie des Sponsors ein Zuwarten bis zur Verifizierung des Verdachts zumutbar erscheint. Basiert die Werbekampagne des Sponsors beispielsweise auf der besonderen Reinheit und Unverfälschtheit seiner Produkte (z.B. bei Lebensmittelherstellern) oder auf seiner besonderen Glaubwürdigkeit (z.B. wenn er hochwertige Dienstleistungen, etwa als Finanzberater, erbringt), kann bereits ein Dopingverdacht beim Gesponserten das Image des Sponsors nachhaltig schädigen. Ist hingegen der Bezug zwischen dem Verstoß des Gesponserten bzw. dem Sponsoringobjekt und den Produkten oder Dienstleistungen des Sponsors inhaltlich weniger stark (wie z.B. in der Regel bei Autoherstellern, Telekommunikations- oder Medienunternehmen), ist ein Zuwarten bis zur Klärung des Verdachts eher zumutbar. Es empfiehlt sich daher, wesentliche Elemente der Werbestrategie des Sponsors im Sponsoringvertrag festzuhalten. Lässt man bereits den qualifizierten Verdacht eines Verstoßes ausreichen, sollten an diesen konkrete und nicht zu niedrige Anforderungen gestellt werden: Bloße Verdächtigungen in der Öffentlichkeit bzw. in

Sponsor nicht zugemutet werden, dass er oder seine Produkte mit dem negativen Image des Dopings behaftet würden.

28 Siehe dazu auch Kratz/ Quantius, Festschrift Fenn, 177, 187 f.; Humberg, JR 2005, 271, $272 \mathrm{f}$. 
den Medien dürften regelmäßig noch keinen hinreichenden Kündigungsgrund abgeben ${ }^{29}$. In Dopingfällen könnte vereinbart werden, dass ein qualifizierter Verdacht bei Vorliegen einer positiven A-Probe gegeben ist ${ }^{30}$ oder wenn sich der Sportler vorgeschriebenen Kontrollen entzieht. Insgesamt sollte eine derartige Vereinbarung wegen der weitreichenden Folgen einer Vertragsbeendigung jedoch nur in Fällen drohender schwerer Imageschäden des Sponsors getroffen werden.

\section{(2) Leistungsstörungen auf Seiten des Gesponserten}

Stellt sich die Verfehlung als Leistungsstörung auf Seiten des Gesponserten dar, kommt ebenfalls eine Kündigung des Vertrags in Betracht. Eine solche Leistungsstörung kann sich insbesondere daraus ergeben, dass der Gesponserte seine Kommunikationsleistung nicht mehr erbringen kann, beispielsweise wenn er wegen Dopings oder Dopingverdachts gesperrt ist und daher nicht mehr bei Wettkämpfen für den Sponsor werben kann. In diesem Sinne sind wohl die Klauseln zu verstehen, die ein außerordentliches Kündigungsrecht des Sponsors vorsehen, wenn der Gesponserte die gesponserten Aktivitäten nicht weiter entfaltet oder seine Kommunikationsleistung nicht erbringt bzw. nicht erbringen kann ${ }^{31}$. Die ausdrückliche Vereinbarung eines derartigen Rechts zur Kündigung wegen Leistungsstörungen auf Seiten des Gesponserten kann sinnvoll sein. Eine solche Regelung dürfte mit allgemeinen vertragsrechtlichen Grundsätzen in Einklang stehen - und damit einer AGB-Kontrolle standhalten, solange sie auf hinreichend schwerwiegende Leistungsstörungen beschränkt ist (etwa unter Berücksichtigung des Rechtsgedankens des $\int 323$ V BGB). Sie kann neben dem Recht zur Kündigung aus wichtigem Grund vor allem dann von Bedeutung sein, wenn die Schwelle des wichtigen Grundes im Einzelfall noch nicht erreicht ist, also noch kein zu starker Imageschaden des Sponsors eingetreten oder zu befürchten ist, er aber keinen adäquaten Gegenwert für seine Sponsorenleistungen mehr erhält.

${ }^{29}$ Siehe dazu auch Kratz/ Quantius, Festschrift Fenn, 177, 188 f.; Humberg, JR 2005, 271, 273; Nesemann, NJW 2007, 2083, 2084 (in Bezug auf Vertragsverstöße allgemein).

30 So für Vertragsverstöße insgesamt auch Nesemann, NJW 2007, 2083, 2084.

31 Siehe z.B. Münchner Vertragshandbuch-Weiand, Bd. 3, Wirtschaftsrecht II, Sportsponsoringvertrag (S. 1237 ff.), \& 8 III lit. b, Weiand/Poser, Sponsoringvertrag, \& 9 III lit. a; ähnlich Beck'sches Formularbuch-Feick, Sponsoringvertrag (S. 1276 ff.), $\int 7 \mathrm{I}$. 
Fraglich ist, ob ein solches Kündigungsrecht auch diejenigen Fälle erfasst, in denen der Gesponserte seine Leistung aus von ihm nicht zu vertretenden Gründen nicht mehr - oder nur noch in geringerem Umfang erbringen kann, etwa wenn - wie bei der Tour de France 2007 - die Fernsehübertragung eines Sportereignisses eingestellt oder verringert wird. Dann sinkt auch die Werbewirkung sämtlicher nicht gedopten Beteiligten. $\mathrm{Ob}$ dies allerdings eine außerordentliche Kündigung gegenüber einem solchen Sportler rechtfertigt, erscheint zweifelhaft. Schadensersatzansprüche dürften hier regelmäßig an mangelndem Verschulden des Gesponserten scheitern. Stattdessen eine außerordentliche Kündigung mit ihren weitreichenden Folgen zuzulassen, erscheint nur denkbar, wenn die Kommunikationsleistung aufgrund der Ereignisse vollständig ausfällt, nicht wenn lediglich ihre Wirkung herabgesetzt ist. Dementsprechend sehen die einschlägigen Vertragsformulare regelmäßig eine fristlose Kündigung nur für diejenigen Fälle vor, in denen sich eine Leistung des Gesponserten insgesamt als undurchführbar erweist, was etwa bei Absage oder Abbruch des gesamten Sportereignisses denkbar erschiene. Auch hier sollte allerdings je nach Gegenstand des Sponsoringvertrags differenziert werden: Bezieht er sich ausschließlich auf das nunmehr abgesagte Sportereignis, wird er gegenstandslos, so dass eine Rückabwicklung angebracht erscheint. Handelt es sich hingegen um einen Sponsoringvertrag, bei dem sich die ausgetauschten Leistungen nicht auf das fragliche Ereignis beschränken, sollte vertraglich geregelt werden, ab welcher Schwelle eine außerordentliche Kündigung wegen Leistungsstörungen auf Seiten des Gesponserten in Betracht kommt. Hier kann bei der Vertragsgestaltung eine Orientierung an den gesetzlichen Regelungen über teilweise Unmöglichkeit (\$\326 V, 323 V 1 BGB) sinnvoll sein.

\section{b) Rechtsfolgen}

Rechtsfolge der außerordentlichen Kündigung ist zunächst die Beendigung des Sponsoringvertrags mit Wirkung ex nunc, sodass für die Zukunft keine weiteren Leistungen erbracht, bereits erbrachte Leistungen aber nicht zurückgewährt werden. Manche Vertragsformulare sehen allerdings für den Fall, dass eine Vertragspartei die fristlose Kündigung zu vertreten hat, eine Pflicht dieser Partei zur Rückgewähr der empfangenen Leistungen bzw. zur 
Erstattung ihres Wertes vor ${ }^{32}$, vereinzelt wird sogar eine von einem Vertretenmüssen der Kündigung unabhängige Rückgewährpflicht beider Beteiligten statuiert ${ }^{33}$. Derartige Verpflichtungen entsprechen nicht dem nach allgemeinen Regeln des Kündigungsrechts zu Erwartenden. Eine einseitige Rückgewähr- bzw. Erstattungspflicht kann daher nur im Sinne eines neben der Kündigung geltend gemachten Schadensersatzanspruchs - etwa nach dem Rechtsgedanken des $₫ 281$ I 2 BGB - verstanden werden ${ }^{34}$. Als Schaden des Sponsors würde dann - gewissermaßen pauschaliert - die erbrachte Sponsorenleistung angesehen. Ob eine solche Pauschalierung allerdings den Grundsätzen des Schadensrechts genügt, erscheint fraglich: Immerhin ist es denkbar, dass der Sponsor in der Vergangenheit einen Gegenwert für seine Leistungen in Form einer Kommunikationsleistung des Gesponserten erhalten und aufgrund der Werbewirksamkeit des Sponsorings Gewinne erwirtschaftet hat. Wird dann gleichwohl die Sponsorenleistung zurückgefordert, erscheint eine solche Pauschalierung vielmehr als eine Art Strafschadensersatz, wie er im deutschen Schadensersatzrecht bislang gerade abgelehnt wird ${ }^{35}$. Daher muss bezweifelt werden, dass derartige Klauseln einer AGB-Kontrolle standhalten würden. Denkbar erscheint sowohl eine Unwirksamkeit nach \309 Nr. 5 BGB in bestimmten Fällen als auch eine Unwirksamkeit nach \307 I, II Nr. 1 BGB wegen Verstoßes gegen wesentliche Grundgedanken der Regelungen über den Schadensersatz wegen Leistungsstörungen. Da die Beteiligten für bestimmte Fälle auch eine Vertragsstrafe vereinbaren können ${ }^{36}$, erscheint eine derartige teilweise Rückabwicklungsregelung auch nicht erforderlich und sollte daher nicht in Sponsoringverträge aufgenommen werden ${ }^{37}$. Dann gelten die allgemeinen

32 Münchener Vertragshandbuch-Weiand, Bd. 3, Wirtschaftsrecht II, Sportsponsoringvertrag (S. 1237 ff.), \8 V; Weiand/Poser, Sponsoringvertrag, \9 V.

33 Beck'sches Formularbuch-Feick, Sponsoringvertrag (S. 1276 ff.), \& 8 S. 1.

${ }^{34}$ Eine Einordnung als Vertragsstrafenversprechen dürfte zum einen daran scheitern, dass die untersuchten Formulare teilweise an anderer Stelle zusätzlich Vertragsstrafenversprechen enthalten (Weiand/ Poser, Sponsoringvertrag, \ 7 V 2), zum anderen daran, dass die Vertragsstrafe nach $\ 339$ BGB nur für den Fall der Nicht- oder Schlechtleistung vereinbart werden darf (siehe Nesemann NJW 2007, 2083, 2084), die wichtigen Gründe für die außerordentliche Kündigung in den Formularen sich jedoch meist nicht auf derartige Leistungsstörungen beschränken.

35 Siehe nur Lange/ Schiemann, Schadensersatz, S. 12 f.

${ }^{36}$ Dazu näher unten V. 2. Selbst bei einer solchen Vereinbarung erscheint es allerdings AGB-rechtlich sowie mit Blick auf $\int 138$ BGB bedenklich, dem Sponsor zu ermöglichen, sämtliche erbrachten Geld- und Sachleistungen zurückzufordern, vgl. Nesemann, NJW 2007, 2083, 2085 f. Auch \343 BGB dürfte in diesem Zusammenhang zu berücksichtigen sein.

37 Teilweise enthalten die Formulare mit derartigen Klauseln auch eine gesonderte Vertragsstrafenregelung (Weiand/ Poser, Sponsoringvertrag, \ 7 II 2). Das spricht 
Vorschriften mit der Folge, dass die fristlose Kündigung des Sponsoringvertrags auch bei schuldhaften Verstößen des Gesponserten die Wirkungen des Vertrags erst für die Zukunft entfallen lässt.

dafür, dass die Vereinbarung im Zusammenhang mit dem Kündigungsrecht nicht als Vertragsstrafe gedeutet werden kann. 



\section{Beendigung aufgrund gesetzlicher Rege- lungen}

Auch ohne gesonderte vertragliche Vereinbarung kann bei Verfehlungen des Gesponserten und damit auch in Dopingfällen eine Beendigung des Sponsoringvertrags aufgrund gesetzlicher Regelungen in Betracht kommen. Voraussetzungen und Rechtsfolgen einer solchen Vertragsbeendigung sind nicht nur für lückenhafte Sponsoringverträge, sondern auch für die AGB-Kontrolle von Rücktrittsklauseln bedeutsam. Zu unterscheiden ist zwischen einer Einordnung der Verfehlung des Gesponserten als Leistungsstörung (1.) und als wichtiger Grund für eine außerordentliche Kündigung des Vertrags (2.). Ferner sind diese Fälle zu denjenigen einer Störung bzw. eines Wegfalls der Geschäftsgrundlage abzugrenzen (3.).

\section{Verfehlung des Gesponserten als Leistungs- störung}

Verfehlungen des Gesponserten, insbesondere Doping, können einen Mangel seiner Kommunikationsleistung darstellen, z.B. wenn personenbezogene Werbung in einem solchen Fall nicht mehr durchführbar ist oder wenn ein Sportler wegen Dopings oder Dopingverdachts gesperrt wird und damit die Werbewirkung seiner Teilnahme an Wettkämpfen entfällt oder seine Werbewirkung insgesamt zumindest erheblich eingeschränkt wird. In derartigen Fällen kommen die für den konkreten Vertrag einschlägigen Mängelgewährleistungsvorschriften zum Zuge. Welche Regelungen das sind, hängt insbesondere vom Charakter der Kommunikationsleistung des Gesponserten ab.

Bei aktiver Werbung des Gesponserten für den Sponsor, die als Werk-

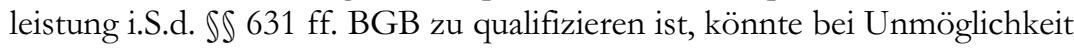


der Werbung (etwa wegen einer Dopingsperre) oder bei Schlechtleistung (etwa weil die Werbung wegen der Verfehlung des Gesponserten weniger Wirkung zeigt) werkvertragliches Mängelgewährleistungsrecht angewandt

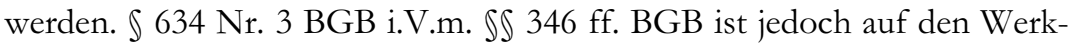
vertrag als Vertragstypus mit einmaligem Leistungsaustausch zugeschnitten und passt daher nicht für Sponsoringverträge, die den Charakter von Dauerschuldverhältnissen haben. Zudem können im Einzelfall Konflikte der werkvertraglichen Regeln mit dem Regelungsregime, das für die Sponsorenleistung gilt, auftreten ${ }^{38}$. In bestimmten Fällen könnte bei werkvertraglicher Einordnung der Leistung des Gesponserten auch eine Kündigung durch den Sponsor aus wichtigem Grund nach $\int 649$ S. 1 BGB in Betracht kommen. Da dieses Kündigungsrecht jedoch nicht auf einer Leistungsstörung basiert, ist es erst im Anschluss zu untersuchen ${ }^{39}$.

Besteht die Kommunikationsleistung des Gesponserten im Verschaffen von Nutzungsmöglichkeiten oder von persönlichkeitsgeprägten Rechtspositionen, kommt als Vertragsbeendigungsregelung in erster Linie $\int 581$ II i.V.m. \543 I, II 1 Nr. 1 BGB in Betracht ${ }^{40}$. Diese Regelung betrifft zwar die außerordentliche fristlose Kündigung aus wichtigem Grund, knüpft jedoch an bestimmte Leistungsstörungen der Vertragspartner an. Ihre Anwendung ist insbesondere dann denkbar, wenn der Gesponserte seine Kommunikationsleistung (etwa wegen einer Dopingsperre) überhaupt nicht erbringen kann ${ }^{41}$; eine vorherige Fristsetzung oder Abmah-

38 So könnte der Sponsor, wenn seine Gegenleistung eine Gebrauchsüberlassung ist, den Vertrag nach $\iint 581$ II, 543 I, II Nr. 3 BGB möglicherweise sofort kündigen, während im Hinblick auf die Werkleistung des Gesponserten dessen Recht auf Nacherfüllung (\$S 634 Nr. 1, 635 BGB) zu beachten wäre. Im Ergebnis müsste hier aber wohl auf die Kommunikationsleistung des Gesponserten als vertragstypische Leistung und damit auf Werkvertragsrecht abgestellt werden.

${ }^{39}$ Unten IV. 2. a.

40 Sofern die Verschaffung persönlichkeitsgeprägter Rechtspositionen im Einzelfall dauerhaft und ausschließlich angelegt ist, wären die Regeln über den Rechtskauf

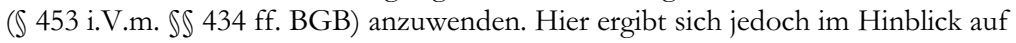
die kaufvertragliche Mängelgewährleistung eine Situation, die im Wesentlichen derjenigen bei der Einordnung der Kommunikationsleistung als Werkleistung entspricht und insbesondere nicht für Sponsoringverträge mit Dauerschuldcharakter passt, sodass diese Variante hier nicht weiter verfolgt wird.

41 SS 581 II, 536a I Var. 1 BGB könnten hingegen allenfalls auf bereits im Zeitpunkt des Vertragsschlusses vorhandene Rechtsmängel der Kommunikationsleistung angewandt werden. Diese Regelungen, deren Heranziehung bei Sponsoringverträgen ohnehin nicht in allen Konstellationen angemessen erscheint (z.B. bei Unklarheiten im Hinblick auf die rechtliche Verwertbarkeit persönlichkeitsgeprägter Rechtspositionen oder bei rechtlichen Hindernissen in Bezug auf die Erteilung einer Lizenz), 
nung dürfte hier regelmäßig nach \543 III Nr. 1 oder 2 BGB entbehrlich sein. Bei bloßer Schlechtleistung, also etwa wenn der Sponsor weiter mit dem Image des Gesponserten werben kann - wenngleich mit geringerem

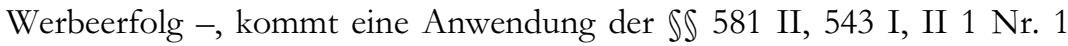
BGB hingegen nicht in Betrach ${ }^{42}$. Rechtsfolge der außerordentlichen fristlosen Kündigung aus wichtigem Grund ist die Beendigung des Vertrags mit Wirkung ex nunc ohne Rückgewähr der bereits erbrachten Leistungen.

Insgesamt zeigt sich, dass mit Hilfe der allgemeinen vertragsrechtlichen Kündigungsmöglichkeiten wegen Leistungsstörungen Schlechtleistungen des Gesponserten nicht hinreichend erfasst werden können. Will man in derartigen Fällen dem Sponsor das Recht einräumen, sich vom Vertrag zu lösen, empfiehlt sich daher eine ausdrückliche Regelung im oben beschriebenen Sinne ${ }^{43}$.

\section{Verfehlung des Gesponserten als wichtiger Grund für eine außerordentliche Kündigung des Ver- trags}

Eine Verfehlung des Gesponserten, wie z.B. Doping, möglicherweise auch schon der Verdacht einer solchen Verfehlung, kann zugleich einen wichtigen Grund für eine außerordentliche Kündigung des Vertrags durch den Sponsor darstellen. Rechtsgrundlage einer solchen außerordentlichen Kündigung aus wichtigem Grund ist in erster Linie \ 314 BGB; sofern die Kommunikationsleistung des Gesponserten als Werkleistung zu beurteilen ist, also bei aktiver Werbung für den Sponsor, kommt auch eine Kündigung nach $₫ 649$ S. 1 BGB in Betracht. Die Anwendbarkeit der speziellen Regelung des \649 BGB ist als Erstes zu untersuchen (a); im Anschluss kann dann beurteilt werden, in welchem Umfang \314 BGB im Verhältnis zu dieser Sonderregelung sowie zu \581 II i.V.m. \543 I, II 1 Nr. 1 BGB noch zum Zuge kommen kann (b).

werden hier ausgeklammert, weil es sich bei Verfehlungen des Gesponserten, insbesondere in Dopingfällen, in der Regel um nach Vertragsschluss eingetretene Leistungsstörungen handelt.

42 Siehe auch Turner, Festgabe Zivilrechtslehrer 1934/35, 669, 681: «Tritt der erwartete sportliche Erfolg nicht ein, so gehört dies zum Risikobereich des Lizenznehmers; vertragliche Ansprüche können daraus nicht abgeleitet werden.»

${ }^{43}$ Oben III. 2. a (2). 
a) Kündigung durch den Sponsor nach \649 BGB

Eine Kündigung durch den Sponsor als Besteller der Werkleistung des Gesponserten nach \649 S. 1 BGB könnte insbesondere dann in Betracht kommen, wenn der Gesponserte aktiv für den Sponsor wirbt und die Gegenleistung des Sponsors in einer Geldleistung besteht, sodass der Vertrag vom Grundtypus her als Werkvertrag anzusehen ist ${ }^{44}$. Das Kündigungsrecht des Sponsors ist von keinen besonderen Voraussetzungen, insbesondere nicht vom Vorliegen eines wichtigen Grundes, abhängig. Allerdings werden die Interessen des Gesponserten dadurch gewahrt, dass er nach $\int 649$ S. 2 BGB trotz der Kündigung die vereinbarte Vergütung verlangen kann und sich lediglich infolge der Aufhebung des Vertrags ersparte Aufwendungen sowie dasjenige, das er durch anderweitige Verwendung seiner Arbeitskraft erwirbt oder zu erwerben böswillig unterlässt, anrechnen lassen muss. Findet der Gesponserte keinen neuen Sponsor - wie etwa regelmäßig in Dopingfällen -, muss der Sponsor dann trotz der Kündigung seine Sponsorenleistung erbringen. \649 BGB ist auf Fallgestaltungen zugeschnitten, in denen der Besteller einer Werkleistung kein Interesse mehr an dieser hat. In diesem Fällen wird ihm - anders als bei sonstigen Austauschgeschäften ${ }^{45}$ - ausnahmsweise ein Kündigungsrecht zugestanden. Diese Regelung passt jedoch nicht für die Situation des Sponsors im Falle einer Verfehlung des Gesponserten, denn hier will der Sponsor den Vertrag nicht aus freier Entscheidung, sondern gerade wegen der Verfehlung des Gesponserten beenden. Daher erscheint eine Anwendung des $\int 649$ BGB hier nicht interessengerecht. Folglich wird durch \649 BGB bei Sponsoringverträgen, die zugleich Dauerschuldverhältnisse darstellen, auch nicht die Anwendung der allgemeinen Kündigungsregelung in $\ 314$ BGB ausgeschlossen.

${ }^{44}$ Besteht die Gegenleistung des Sponsors hingegen in einer Sachleistung oder Tätigkeit, erscheint die Anwendung des $₫ 649$ BGB von vornherein nicht interessengerecht, weil \649 S. 2 BGB hier dem Gesponserten häufig keine hinreichende Substitution der Sponsorenleistung ermöglichen dürfte, etwa weil er entsprechende Sach- oder Dienstleistungen von anderer Seite möglicherweise gar nicht oder nur zu einem höheren Preis erhalten kann.

45 Siehe auch Staudinger-Peters \649 Rn. 7; MünchKommBGB-Busche \649 Rn. 1 f.; Prütting/ Wegen/ Weinreich-Leupertz, \649 Rn. 1 (für Austauschgeschäfte eigentlich systemwidriger Gedanke). 
b) Kündigung durch den Sponsor nach \ 314 BGB

\section{(1) Verbältnis zu anderen Kündigungsregelungen}

Ist $\int 314 \mathrm{BGB}$ also gegebenenfalls neben $\int 649 \mathrm{BGB}$ anwendbar, bleibt noch das Verhältnis des $\int 314$ BGB zu \581 II i.V.m. \543 I, II 1 Nr. 1 BGB zu klären. Der Unterschied ist bedeutsam, weil die spezielle Vertragsbeendigungsregelung bei der Rechtspacht von den Verfehlungen des Gesponserten nur diejenigen erfasst, bei denen seine Kommunikationsleistung über einen längeren Zeitraum überhaupt nicht mehr erbracht wird. Die Schwelle für die Anwendung dieser Regelung ist also relativ hoch, sodass zu fragen ist, ob neben dieser Spezialregelung $\ 314$ BGB anwendbar ist, der unter Umständen bereits bei einmaliger Nichtleistung oder auch nur Schlechtleistung des Gesponserten (etwa bei nur noch geringer verbleibender - oder gar «negativen» - Werbewirkung) eingreifen kann. Hierbei ist zu berücksichtigen, dass $\int 543$ BGB in erster Linie auf Mietverträge und damit auf die Gebrauchsüberlassung von Sachen gegen Entgelt zugeschnitten ist. Schon für die Rechtspacht ist die Anwendung dieser Regelung in Ermangelung speziellerer Vorschriften für die Einräumung von Rechten eher eine «Hilfskonstruktion«, und der Sponsoringvertrag, der in der Regel nicht als «reine» Rechtspacht, sondern allenfalls als gemischter Vertrag mit Elementen der Rechtspacht anzusehen ist, ist von der Ausgangssituation des $\int 543$ BGB noch weiter entfernt. Angesichts der Vielzahl denkbarer Leistungen, die bei einem Sponsoringvertrag mit Elementen der Rechtspacht zusammentreffen können, erscheint daher jedenfalls ein Vorrang des $\int 543$ BGB gegenüber allgemeinen Regelungen, die eine flexible Berücksichtigung aller Interessen im Einzelfall ermöglichen, nicht angemessen. Daher ist $\int 314$ BGB auch bei Sponsoringverträgen mit einer Kommunikationsleistung, die nach den Regeln der Rechtspacht zu beurteilen ist, anzuwenden. Ob daneben im Einzelfall eine Kündigung nach $\ 581$ II i.V.m. $\int 543$ I, II 1 Nr. 1 BGB in Betracht kommt, kann hier offen bleiben, da in den untersuchten Fällen von Verfehlungen des Gesponserten eine Anwendung des $₫ 314$ BGB jedenfalls keine höheren Anforderungen an die Kündigung stellt als $\ 581$ II i.V.m. \ 543 I, II 1 Nr. 1 BGB, sodass der Sponsor wohl regelmäßig nach $₫ 314$ BGB vorgehen wird. 
(2) Voraussetzungen einer Kündigung nach \314 BGB

Ein Dauerschuldverhältnis kann nach \314 I BGB aus wichtigem Grund gekündigt werden, wenn dem Kündigenden unter Berücksichtigung aller Umstände des Einzelfalls und unter Abwägung der beiderseitigen Interessen die Fortsetzung des Vertragsverhältnisses bis zur vereinbarten Beendigung oder bis zum Ablauf einer Kündigungsfrist nicht zugemutet werden kann. Ein wichtiger Grund in diesem Sinne kann auch eine Verfehlung des Vertragspartners, wie etwa Doping eines gesponserten Sportlers, sein. Insoweit gilt das bereits im Zusammenhang mit dem vertraglich vereinbarten Recht zur Kündigung aus wichtigem Grund näher Ausgeführte ${ }^{46}$ : Ein wichtiger Grund dürfte in der Regel vorliegen beim Verstoß gegen gesetzliche Vorschriften, aber wohl auch - wegen des Imagetransfers als wesentlichem Ziel des Sponsoringvertrags - beim Verstoß gegen für den Sportler verbindliche Regelwerke ${ }^{47}$. Hingegen dürfte eine bloße «Verschlechterung» der Kommunikationsleistung des Gesponserten, etwa wegen einer Beendigung oder Kürzung der Fernsehberichterstattung wegen Dopingfällen, an denen der Gesponserte selbst nicht beteiligt ist, regelmäßig noch keinen wichtigen Grund darstellen, der eine außerordentliche Kündigung rechtfertigt. Die Schwelle für die Entstehung des Kündigungsrechts dürfte hier erst beim vollständigen Ausfall der Kommunikationsleistung des Gesponserten erreicht sein. Dann aber sind auch Abmahnung bzw. Setzen einer Abhilfefrist nach \314 II BGB entbehrlich.

Auch in Bezug auf das Hinreichen des qualifizierten Verdachts eines solchen Verstoßes gelten die oben entwickelten Leitlinien, d.h. es kommt insbesondere darauf an, ob der Imagetransfer als wesentliches Vertragsziel bereits beim Verdacht eines Verstoßes in Frage gestellt ist. Fehlt eine diesbezügliche Vereinbarung im Vertrag, dürfte eine außerordentliche Kündigung wegen eines qualifizierten Dopingverdachts nur unter engen Voraussetzungen in Betracht kommen. Diese sind ähnlich zu bestimmen wie bei den oben empfohlenen vertraglichen Regelungen, sodass konkrete Anhaltspunkte für Doping (wie insbesondere eine positive A-Probe oder eine Umgehung von Kontrollen) vorliegen müssen und ein schwerer Imageschaden des Sponsors zu befürchten ist. Entscheidend ist, ob es dem Sponsor bereits nicht mehr zugemutet werden kann, bis zur Klärung des Verdachts am Vertrag festzuhalten, ob also die auch schon im Verdachts-

\footnotetext{
46 S.o. III. 2. a (1).

47 Vgl. auch Bergermann, Doping und Zivilrecht, S. 239, der das Verbot des Dopings als

Inhalt des Sponsoringvertrags ansieht.
} 
fall denkbaren Schadensersatzansprüche ${ }^{48}$ allein den Interessen des Sponsors nicht hinreichend Rechnung tragen können. Die Situation ist hier anders als im Verbandsrecht, wo in derartigen Verdachtsfällen mitunter auf den Anscheinsbeweis ${ }^{49}$ bzw. - vor allem im internationalen Bereich - auf das Prinzip der «strict liability» ${ }^{50}$ zurückgegriffen wird, denn wegen der hohen Bedeutung des Imagetransfers beim Sponsoring kann die Rücktrittsschwelle hier niedriger liegen als die Schwelle für die Verhängung von Verbandsstrafen. Allerdings kann hier nach \314 II BGB zunächst eine Abmahnung erforderlich sein, um dem Gesponserten die Möglichkeit zu geben, den gegen ihn bestehenden qualifizierten Dopingverdacht auszuräumen. Hier ist im konkreten Fall abzuwägen, ob eine realistische Möglichkeit besteht, den Verdacht auszuräumen (z.B. wenn der Verdacht lediglich auf dem Versäumen einer Dopingkontrolle beruht) und ob der bereits eingetretene oder drohende Imageschaden des Sponsors ein Zuwarten bis zum Ablauf einer solchen Abhilfefrist zumutbar erscheinen lässt ( $\$ 314$ II 2 i.V.m. \323 II Nr. 3 BGB).

Ein wichtiger Grund kann weiterhin damit begründet werden, dass der Gesponserte seine vertraglich geschuldete Leistung nicht oder nicht ordnungsgemäß erbringt, also insbesondere wenn ein Sportler wegen einer Dopingsperre seine Kommunikationsleistung nicht erbringen kann. Die in solchen Fällen nach \314 II 1 BGB eigentlich erforderliche Fristsetzung bzw. Abmahnung dürfte hier regelmäßig nach \314 II 2 i.V.m. \323 II Nr. 3 BGB entbehrlich sein.

Wie schnell der Sponsor reagieren muss, um innerhalb einer angemessenen Frist i.S.d. \ 314 III BGB zu kündigen, hängt von den Umständen des Einzelfalls ab. Steht die Verfehlung des Gesponserten fest, dürfte dem Sponsor regelmäßig keine allzu lange Frist mehr zugebilligt werden; zögert er hier, liegt der Gedanke nahe, dass sein drohender Imageschaden doch nicht so gravierend ist, dass ihm ein wichtiger Grund für die außerordentliche Kündigung zur Seite steht. Besteht lediglich der Verdacht einer Verfehlung des Gesponserten, dürfte dem Sponsor hingegen eine längere Überlegungs- und Prüfungsfrist zugestanden werden. Kündigt er hier zu

\footnotetext{
48 Dazu näher unten V. 1.

49 Siehe z.B. OLG Frankfurt NJW-RR 2000, 1117, 1121; ähnlich OLG Düsseldorf NJW-RR 1996, 696, 697; Horst/ Jacobs, RdA 2003, 215, 216; Walker, in: Vieweg (Hrsg.), Doping. Realität und Recht, S. 144 f.

${ }^{50}$ Dazu z.B. Netzle, in: Vieweg (Hrsg.), Doping. Realität und Recht, S. 197, 206 ff.; Horst/Jacobs, RdA 2003, 215, 216.
} 
Unrecht, hätte dies für den Gesponserten und dann - wegen etwaiger Schadensersatzpflichten - möglicherweise auch für den Sponsor selbst gravierende Auswirkungen. Daher sollte hier im Interesse beider Vertragspartner regelmäßig von einer längeren Kündigungsfrist, die es zumindest ermöglicht, dem Verdacht nachzugehen und die Rechtslage zu überdenken $^{51}$, ausgegangen werden.

Eine Kündigung nach $\int 314$ BGB kommt nach allgemeinen Grundsätzen (Verbot des venire contra factum proprium, $\int 242$ BGB) nicht in Betracht, wenn der Sponsor für die Verfehlung des Gesponserten selbst verantwortlich ist, insbesondere weil er ihn zum Doping veranlasst oder es ihm zumindest nahe gelegt hat ${ }^{52}$. Auch bei bloßer Kenntnis des Sponsors vom Doping des Gesponserten dürfte die Möglichkeit zur außerordentlichen Kündigung aus wichtigem Grund entfallen ${ }^{53}$. Ebenso erschiene es treuwidrig, wenn ein Sponsor den Sponsoringvertrag in einem Dopingfall kündigen wollte, wenn er seine mit dem Sponsoringvertrag verfolgten kommunikativen Ziele, beispielsweise einen Bekanntheitsgrad von $100 \%$ auf dem von ihm anvisierten Markt, bereits vor dem Ende der regulären Vertragslaufzeit erreicht hat. Hier dürften in erster Linie Schadensersatzansprüche wegen späterer Verluste aufgrund dopingbedingter Imageeinbußen in Betracht kommen.

Diskussionsbedürftig ist ferner die Fallkonstellation, in der Partner des Sponsoringvertrags nicht der gedopte Sportler selbst ist, sondern der Verein oder Verband, dem dieser Sportler angehört, und den Verein oder Verband keine Verantwortung für das Doping trifft. Da die Kündigung verschuldensunabhängig ist, kommt eine Zurechnung des Handelns des Sportlers nach $\int 278 \mathrm{BGB}$ nicht in Betracht ${ }^{54}$ und eine Heranziehung von $\int 31$ BGB scheitert regelmäßig daran, dass der Sportler kein Organ des Vereins im Sinne dieser Vorschrift ist ${ }^{55}$. Man könnte erwägen, eine Kenntnis des Sportlers vom Doping analog \166 I BGB auch dem Verein zuzurechnen. Es erscheint allerdings bereits zweifelhaft, ob der Sportler im

${ }^{51}$ Eine vollständige rechtliche Klärung dürfte angesichts der erforderlichen einzelfall-

bezogenen Interessenabwägung regelmäßig nicht in Betracht kommen.

52 Vgl. auch Kraty/ Quantius, Festschrift Fenn, 177, 189 f.; Humberg, JR 2005, 271, 273.

53 Ebenso Kratz/ Quantius, FS Fenn, 177, 189 f.; Humberg, JR 2005, 271, 273.

${ }^{54}$ Kraty/ Quantius, Festschrift Fenn, 177, 191 Fn. 69; zu pauschal (ohne Differenzierung zwischen Rücktritt und Schadensersatz) hingegen Turner, Festgabe Zivilrechtslehrer 1934/35, 669, 681; Bergermann, Doping und Zivilrecht, S. 244.

${ }^{55}$ Kratz/ Quantius, Festschrift Fenn, 177, 191 Fn. 69; Humberg, JR 2005, 271, 273. 
Verhältnis zum Verein angesichts zahlreicher verbindlicher Regelwerke auf Vereins- und Verbandsebene eine so selbständige Stellung innehat, dass eine Wissenszurechnung nach $\int 166$ BGB gerechtfertigt wäre. Zudem setzt das Vorliegen eines wichtigen Grundes i.S.d. \314 BGB keine Kenntnis des Kündigungsgegners voraus, und wo kein Wissen erforderlich ist, kann auch keines zugerechnet werden. Daher ist eine Zurechnung keine Voraussetzung der außerordentlichen Kündigung nach $₫ 314$ BGB. Vielmehr ist bei der Prüfung, ob ein wichtiger Grund vorliegt, der eine außerordentliche Kündigung rechtfertigen könnte, zu berücksichtigen, ob die Verfehlung letztlich in den Risikobereich des Vertragspartners des Sponsors fällt. Weiterhin ist in Rechnung zu stellen, dass es sich hier regelmäßig nicht um eine vollständige Schlecht- bzw. Nichtleistung handelt. Dafür sollte der Rechtsgedanke des $₫ 323 \mathrm{~V}$ BGB entsprechend herangezogen werden ${ }^{56}$, weil \314 BGB insoweit eine Regelungslücke aufweist und die Interessenlage vergleichbar ist. Für die Frage, ob ein wichtiger Grund i.S.d. \314 I BGB vorliegt, wird bislang insbesondere auf die Bedeutung des Sportlers innerhalb des Vereins abgestellt: Sofern gerade der gedopte Sportler das Image des Vereins entscheidend prägt, seien die Auswirkungen für den Sponsor als mit denjenigen beim unmittelbaren Sponsoring des Gedopten vergleichbar anzusehen; im Einzelfall komme es entscheidend auf die Stellung des Gedopten innerhalb des Vereins oder Verbandes an ${ }^{57}$. Das erscheint vom Ansatzpunkt her richtig, allerdings dürfte die Schwelle für das Vorliegen eines wichtigen Grundes hier insgesamt höher liegen als bei einem Vertrag mit dem Gedopten selbst: Auf einen Verstoß gegen gesetzliche Vorschriften wird die Kündigung gegenüber dem Verein oder Verband vor allem dann gestützt werden können, wenn dieser vom Doping wusste oder gar aktiv daran mitgewirkt hat. Bei Unkenntnis des Vereins oder Verbandes kann die Kündigung möglicherweise mit einem Verstoß gegen vertragliche Vereinbarungen begründet werden, insbesondere wenn der Verein oder Verband Dopingfreiheit seiner Mitglieder vertraglich garantiert hat. Andernfalls kommt als Kündigungsgrund vor allem der Imageverlust des Sponsors oder eine Schlechtleistung in Bezug auf die Kommunikationsleistung des Gesponserten in Betracht. In diesen beiden Fällen ist zu berücksichtigen, dass hier das Gewicht des Imageverlusts bzw. der Schlechtleistung innerhalb des gesamten Vertrags ein anderes ist als beim Sponsoringvertrag mit dem Gedopten selbst. Daher muss das Gewicht des Verstoßes, also insbesondere der Einfluss des Dopingfalles auf den gesamten Verein

\footnotetext{
56 Siehe auch MünchKommBGB-Gaier, \314 Rn. 11; Prütting/ Wegen/ WeinreichMedicus, \ 314 Rn. 11 (Heranziehung von \ 323 V 2 BGB).

${ }^{57}$ Kratz/ Quantius, Festschrift Fenn, 177, 191 f.; Humberg, JR 2005, 271, 273.
} 
oder Verband, erheblich sein, um einen wichtigen Grund zur außerordentlichen Kündigung des Sponsoringvertrags (als ultima ratio gegenüber anderen Sekundäransprüchen) abzugeben. Dabei ist auch zu berücksichtigen, dass das Andauern bzw. die Verlässlichkeit eines Sponsoringengagements als finanzielle Basis eine wichtige Voraussetzung für den Erhalt von Wettkampflizenzen sein kann. In der Literatur wird vereinzelt argumentiert, dass ein Verein eine Kündigung durch den Sponsor durch Entlassung des Gedopten möglicherweise abwenden könne ${ }^{58}$. Das erscheint angesichts der anderen verbleibenden Rechtsbehelfe als wichtiger Gesichtspunkt. Allerdings haben beispielsweise die Ereignisse im Zusammenhang mit der Tour de France gezeigt, dass manchmal selbst eine solche Maßnahme den Imageverlust des Vereins bzw. Verbands und damit regelmäßig auch des Sponsors nicht abwenden kann, etwa wenn die Glaubwürdigkeit einer Sportart insgesamt durch die Dopingfälle leidet. Dann muss unter Berufung auf den ausbleibenden bzw. sich möglicherweise sogar ins Negative verkehrenden Imagetransfer auch eine außerordentliche Kündigung gegenüber einem Verein oder Verband nach \314 BGB möglich bleiben.

\section{(3) Rechtsfolgen einer Kündigung nach $\int 314$ BGB}

Die außerordentliche Kündigung nach $\int 314$ BGB beendet den Sponsoringvertrag mit Wirkung ex nunc, d.h. für die Zukunft werden keine weiteren Leistungen erbracht, bereits erbrachte Leistungen werden nicht zurückgewährt. Verbleibt beim Sponsor trotz des Rücktritts noch ein Schaden, kann dieser nach $\ 314$ IV BGB in Verbindung mit der jeweils einschlägigen Anspruchsgrundlage für Schadensersatz zusätzlich geltend gemacht werden.

\section{Abgrenzung zu Störung bzw. Wegfall der Ge- schäftsgrundlage, $\mathbb{} 313$ BGB}

Denkbar erschiene es auf den ersten Blick auch, ein «tadelloses» Verhalten des Gesponserten als Geschäftsgrundlage des Sponsoringvertrags anzusehen und dann für die Vertragsbeendigung \ 313 III BGB heranzuziehen ${ }^{59}$. Fraglich ist aber, ob ein Ausweichen auf dieses gegenüber Spezialregelun-

${ }^{58}$ Kraty/ Quantius, Festschrift Fenn, 177, 192.

${ }^{59}$ So - in Bezug auf das österreichische Recht - etwa Holzer/ Fritzweiler, in: Fritzweiler

(Hrsg.), Doping. Sanktionen Beweise Ansprüche, S. 57, 73. 
gen grundsätzlich nachrangige ${ }^{60}$ Rechtsinstitut für die hier untersuchten Fälle erforderlich ist. Wie gezeigt, lassen sich Verfehlungen des Gesponserten mit Hilfe der gesetzlichen Vertragsbeendigungsregeln, insbesondere durch $\int 314$ BGB, hinreichend erfassen. Hier verbleibt auch ein hinreichender Spielraum zur Berücksichtigung der Umstände des konkreten Falles, so dass eine - ergänzende oder gar alternative - Anwendung des $\S 313$ BGB in Bezug auf die Vertragsbeendigung nicht erforderlich erscheint. Soll der Vertrag wegen der Verfehlung des Gesponserten nicht beendet, sondern allenfalls modifiziert werden, ist eine Heranziehung des \ 313 BGB ebenfalls nicht notwendig, denn hier lassen sich über die allgemeinen Leistungsstörungsregeln angemessene Ergebnisse, insbesondere eine Herabsetzung der Sponsorenleistung, erreichen ${ }^{61}$.

60 Siehe dazu hier nur MünchKommBGB-Roth, \313 Rn. 114, 134; Prütting/ Wegen/ Weinreich-Medicus, $\int 313$ Rn. 4.

${ }^{61}$ Dazu näher Schaub, Sponsoring und andere Verträge zur Förderung überindividueller Zwecke, S. 456 f., 461 f. 



\section{Alternative vertragsrechtliche Möglichkeiten}

Neben dem Rücktritt verbleiben dem Sponsor bei Verfehlungen des Gesponserten alternative vertragsrechtliche Möglichkeiten, die im Folgenden kurz dargestellt werden sollen. In Betracht kommen insbesondere Schadensersatzansprüche sowie die Vereinbarung einer Vertragsstrafe.

\section{Schadensersatzansprüche}

$\int 314$ IV BGB stellt klar, dass die Kündigung Schadensersatzansprüche nicht ausschließt. Solche Ansprüche kommen für die bis zur Vertragsbeendigung entstandenen Schäden in Betracht und können sich aus allgemeinen schuldrechtlichen Regeln ergeben, etwa wegen Leistungsstörungen bei der Kommunikationsleistung des Gesponserten oder wegen Verletzung vertraglicher Pflichten (einschließlich der Pflicht, sich an sportliche Regelwerke, die Doping verbieten, zu halten), möglicherweise auch wegen Betrugs gegenüber dem Sponsor. Entscheidend für die Rechtsgrundlage des Schadensersatzanspruchs ist wiederum die vertragsrechtliche Einordnung der Pflichten der Beteiligten, insbesondere der Kommunikationsleistung des Gesponserten, im konkreten Fall. Deliktsrechtliche Ansprüche des Sponsors dürften daneben eine eher untergeordnete Rolle spielen: Ansprüche aus $\int 823$ II BGB in Verbindung mit möglicherweise durch den Gesponserten verwirklichten Straftatbeständen kommen ohnehin nur in Betracht, wenn die Straftatbestände gerade dem Schutz des Sponsors dienen sollten, was wohl allenfalls bei $₫ 263$ StGB in Betracht kommen dürfte, und selbst wenn sich Doping im Einzelfall einmal als vorsätzliche sittenwidrige Schädigung des Sponsors darstellen sollte - was allenfalls unter besonderen Umständen in Betracht kommen dürfte ${ }^{62}$-, wird ein Anspruch des Sponsors aus $\int 826$ BGB regelmäßig nicht weiter reichen als vertragsrechtliche Schadensersatzansprüche.

${ }^{62}$ Sehr weitgehend Deutsch, VersR 2008, 145, 150 f. 
Schadensersatzansprüche sind für das Kündigungsrecht des Sponsors in zweierlei Hinsicht von Bedeutung: Zum einen ist bei der Überprüfung, ob die Verfehlung des Gesponserten schwer genug ist, um einen wichtigen Grund für eine außerordentliche Kündigung abzugeben, zu berücksichtigen, ob den Interessen des Sponsors bereits durch die Geltendmachung von Schadensersatzansprüchen und ein Zuwarten bis zum nächsten ordentlichen Kündigungstermin hinreichend Rechnung getragen werden kann. Zum anderen kann durch Schadensersatzansprüche derjenige Schaden des Sponsors erfasst werden, der bis zur Vertragsbeendigung eingetreten ist. Das kann beispielsweise ein sofort mit dem Bekanntwerden eines Dopingfalles in Verbindung stehender Umsatzrückgang des Sponsors sein; der dem Sponsor obliegende Nachweis der Kausalität zwischen der Verfehlung des Gesponserten und seinen Verlusten dürfte in diesen Fällen allerdings häufig praktische Probleme aufwerfen.

\section{Vertragsstrafe}

Diesen Schwierigkeiten kann begegnet werden, wenn die Beteiligten von vornherein für bestimmte Verfehlungen des Gesponserten, wie insbesondere Doping, ein Vertragsstrafenversprechen in den Vertrag aufnehmen ${ }^{63}$. Fraglich ist, ob eine solche Vereinbarung eine fristlose Kündigung ausschließt, wenn der wichtige Grund für die Kündigung in derselben Verfehlung besteht. Hier könnte angenommen werden, dass die Beteiligten für derartige Fälle gerade keine Vertragsbeendigung vorgesehen, sondern vielmehr die Vertragsstrafe als angemessene - und dann auch ausreichende Sanktion angesehen haben. Andererseits ist zu bedenken, dass ein denkbarer Zweck der Vertragsstrafe auch sein kann, dem Gläubiger den Schadensnachweis zu ersparen ${ }^{64}$. Das kann auch für Schadensersatzansprüche für die Zeit vor der Beendigung des Sponsoringvertrags von Bedeutung sein und kollidiert dann nicht mit der Vertragsbeendigung. Ob eine Vertragsstrafe nur solche oder aber sämtliche Schäden des Sponsors erfasst, ist im Einzelfall freilich schwer feststellbar. Rückschlüsse lassen sich allenfalls aus der Höhe der Vertragsstrafe ziehen, was allerdings im konkreten Fall nicht einfach sein dürfte. Für das Verhältnis zur Kündigung aus wichtigem Grund lässt sich folgern, dass mit zunehmender Höhe einer Vertragsstrafe verstärkt zu untersuchen ist, ob die Beteiligten damit nicht sämtliche Fol-

${ }^{63}$ Dazu ausführlich Nesemann, NJW 2007, 2083, 2084 ff.; weiterhin - in Bezug auf Arbeitsverträge - insb. Walker, Festschrift Röhricht, 1277, 1281 f., 1289 ff.

${ }^{64}$ Siehe z.B. BGHZ 63, 256, 259; 85, 305, 313; 105, 24, 27; Nesemann, NJW 2007, 2083, 2084. 
gen eines Verstoßes abdecken wollten, sodass eine Kündigung aus wichtigem Grund, die auf der gleichen Verfehlung wie die Vertragsstrafe beruht, ausgeschlossen ist. 



\section{Fazit}

Es hat sich gezeigt, dass bei Verfehlungen des Gesponserten, wie etwa bei Doping, sowohl aufgrund vertraglicher Regelungen als auch - bei Fehlen entsprechender Vereinbarungen - kraft Gesetzes insbesondere eine außerordentliche Kündigung durch den Sponsor aus wichtigem Grund in Betracht kommt. Die Möglichkeiten einer Kündigung wegen Leistungsstörungen bei der Kommunikationsleistung des Gesponserten haben daneben in erster Linie Bedeutung für Fälle, in denen der Imageschaden des Sponsors nicht allzu gravierend ist. In Bezug auf die Voraussetzungen der auBerordentlichen Kündigung besteht bei den typischen einzelvertraglichen Klauseln und einer Kündigung nach gesetzlichen Regelungen weitgehende Parallelität: In Dopingfällen ist zunächst zu prüfen, ob dem Sponsor ein Zuwarten bis zum nächsten ordentlichen Kündigungstermin zugemutet werden kann. Ist dies nicht der Fall, muss weiter untersucht werden, ob die Kündigung als ultima ratio im konkreten Fall angemessen ist, was insbesondere von der Schwere der Verfehlung (positiv nachgewiesenes Doping oder lediglich Verdacht) abhängt, weiterhin von der Person des Vertragspartners (Gedopter selbst oder Verein bzw. Verband). Dabei ist auch zu berücksichtigen, ob den Interessen des Sponsors möglicherweise bereits durch die Geltendmachung von Schadensersatzansprüchen oder durch eine Vertragsstrafe hinreichend Rechnung getragen werden kann.

Für die Vertragsgestaltung empfiehlt es sich - neben einer Regelung über die ordentliche Kündigung, die bei längerfristigen Verträgen selbstverständlich sein sollte -, die denkbaren Verfehlungen des Gesponserten, wegen denen eine außerordentliche Kündigung aus wichtigem Grund in Betracht kommen soll, konkret festzulegen. Für Dopingfälle erscheint eine Verweisung auf die Voraussetzungen des Dopings in für den Gesponserten maßgeblichen verbandsrechtlichen Regelwerken sinnvoll. Sofern bereits im Falle eines Dopingverdachts gekündigt werden soll, sollten die 
Voraussetzungen eines solchen Verdachts im Vertrag konkretisiert werden; mit Blick auf die weitreichenden Folgen der Vertragsbeendigung sollte die Verdachtsschwelle hier jedoch nicht zu niedrig angesetzt werden. Empfehlenswert erscheint auch eine Klarstellung des Zusammenhangs zwischen der konkreten Werbestrategie des Sponsors und der Kommunikationsleistung des Gesponserten, um die Vertragsauslegung bei von den Beteiligten nicht konkret antizipierten Verfehlungen des Gesponserten zu erleichtern. In Bezug auf die Rechtsfolgen der außerordentlichen Kündigung sind regelmäßig keine besonderen vertraglichen Regelungen erforderlich. Will man gleichwohl eine ausdrückliche Rechtsfolgenregelung treffen, sollte die Vertragsbeendigung ex nunc mit Wegfall der beiderseitigen Leistungspflichten für die Zukunft im Vertrag festgelegt werden. Weitergehende Regelungen, insbesondere die Festlegung einer einseitigen Rückgewährpflicht, erscheinen wegen der Möglichkeit eines zusätzlichen Schadensersatzanspruchs oder ggf. einer Vertragsstrafe nicht erforderlich und zumindest in Allgemeinen Geschäftsbedingungen bedenklich. Zusätzliche Vereinbarungen im Hinblick auf Schadensersatzansprüche bzw. Vertragsstrafen sind denkbar; sie können insbesondere zu Klarstellungszwecken sinnvoll sein. Zu beachten ist aber, dass sie nicht zu einer Überkompensation des Sponsors führen dürfen.

Eine Regelung über die Kündigung aus wichtigem Grund mit Bezug auf Dopingfälle sollte daher insbesondere folgende sponsoringspezifische Komponenten enthalten ${ }^{65}$ :

- Einen Hinweis darauf, dass das Recht zur außerordentlichen Kündigung aus wichtigem Grund (für beide Beteiligten) unberührt bleibt oder eine ausdrückliche Festlegung, dass jede Vertragspartei berechtigt ist, den Vertrag aus wichtigem Grund fristlos zu kündigen.

- Eine beispielhafte Konkretisierung der denkbaren Verfehlungen des Gesponserten, die als wichtige Gründe anzusehen sind. Das können sowohl Verstöße gegen für den Gesponserten maßgebliche sportrechtliche Regelwerke (vereins- und verbandsrechtliche Regeln, Regeln von DSB, NADA, WADA etc.) sein als auch Verstöße gegen vertraglich festgelegte Grundsätze in Bezug auf die Kommunikationsleistung des

${ }^{65}$ Nicht erörtert werden hier die sonstigen, nicht sponsoringspezifischen Komponenten, wie z.B. Festlegungen über die Form der Kündigung oder Bestimmungen über eine außergerichtliche Streitbeilegung bei Uneinigkeit über das Vorliegen der Kündigungsvoraussetzungen. 
Gesponserten (z.B. Konformität des Verhaltens des Gesponserten mit der Werbestrategie des Sponsors, die - an anderer Stelle des Vertrags - genauer zu umschreiben wäre), weiterhin auch der Ausfall oder die Verschlechterung (insbesondere die umfangmäßige Einschränkung) der Kommunikationsleistung des Gesponserten.

- Sofern eine Kündigungsmöglichkeit bereits im Falle eines qualifizierten Verdachts von Verfehlungen des Gesponserten angestrebt wird, sollte dies ausdrücklich vertraglich geregelt und vor allem festgelegt werden, welche Umstände einen solchen qualifizierten Verdacht begründen.

- Eine Klarstellung, dass Schadensersatzansprüche unberührt bleiben.

- Im Falle der zusätzlichen Vereinbarung einer Vertragsstrafe eine Klarstellung, in welchem Verhältnis diese zum Recht auf außerordentliche Kündigung stehen soll. Wenn beide dieselbe Verfehlung betreffen, sollte bei der Festsetzung der Höhe der Vertragsstrafe bedacht werden, ob daneben noch eine Kündigung aus wichtigem Grund möglich bleiben soll.

- Nicht zwingend erforderlich sind Regelungen über Abmahnung oder Fristsetzung vor der außerordentlichen Kündigung sowie über die Rechtsfolgen der außerordentlichen Kündigung, denn hier kann insbesondere $\int 314$ BGB herangezogen werden. Will man gleichwohl eine Regelung treffen, sollte diese eng an den Wortlaut des $₫ 314$ (i.V.m. \323 II) BGB angelehnt werden, denn dort sind die maßgeblichen Abwägungskriterien enthalten und eine konkretere Regelung dürfte im Einzelfall kaum möglich sein, will man nicht für jede denkbare Verfehlung des Gesponserten vorab eine Einzelfallabwägung treffen, was kaum praktikabel erscheint.

Mit Hilfe einer derartigen Vertragsgestaltung sollte es möglich sein, die Besonderheiten von Verfehlungen des Gesponserten, insbesondere von Doping, in Sponsoringverträgen hinreichend zu erfassen und dabei die Interessen beider Seiten angemessen zu berücksichtigen. 



\section{Literatur}

Adolphsen, Jens, Anforderungen an Dopingstrafen nationaler Sportverbände, SpuRt 2000, 97 - 101

Bannenberg, Britta, Das neue «Anti-Doping-Gesetz» hilft dem Sport nicht, SpuRt 2007, 155 - 156

Bergermann, Michael, Doping und Zivilrecht. Rechtmäßigkeit von Doping-Sanktionen sowie durch Doping begründete Schadensersatzansprüche, Frankfurt a.M. 2002

Bruggmann, Thomas/ Grau, Kevin J., Das schmutzige Herz des Sports: Doping und die Haftungsrisiken der Pharmaunternehmen, PharmR 2008, 101 - 108

Brubn, Manfred/ Meblinger, Rudolf, Rechtliche Gestaltung des Sponsoring, Bd. I: Allgemeiner Teil, 2. Aufl. München 1995

Cherkeh, Rainer Tarek, Betrug ( $\$ 263$ StGB), verübt durch Doping, Frankfurt a.M. 2000

Cherkeh, Rainer Tarek/ Momsen, Carsten, Doping als Wettbewerbsverzerrung?, NJW 2001, 1745 - 1752

Deutsch, Erwin, Doping als pharmarechtliches und zivilrechtliches Problem, VersR 2008, 145 - 151

Fischer, Michael, Die Unentgeltlichkeit im Zivilrecht, Köln/ Berlin/ Bonn/ München 2002

Friedrich, Walther, Doping und zivilrechtliche Haftung, SpuRt 1995, 8 11

Fritzweiler, Jochen, Ein \299a StGB als neuer Straftatbestand für den sich dopenden Sportler?, SpuRt 1998, 234 - 235

Fritzweiler, Jochen/ Pfister, Bernhard/ Summerer, Thomas, Praxishandbuch Sportrecht, 2. Aufl. München 2007

Glauben, Paul, Nicht jede Unsportlichkeit ist strafbar, DRiZ 2006, 308 $-309$ 
Grotr, Stefan, Zur Betrugsstrafbarkeit des gesponsorten und gedopten Sportlers, SpuRt 2005, $93-97$

Gutheil, Ulrike, Doping. Die haftungsrechtliche und sportrechtliche Verantwortung von Sportler, Trainer, Arzt und Sportverband, Hamburg 1996

Hauptmann, Markus/ Rübenstabl, Markus, Zur Doping-

Besitzstrafbarkeit des Sportlers de lege lata und de lege ferenda, MedR 2007, 271 - 279

Heger, Martin, Die Strafbarkeit von Doping nach dem Arzneimittelgesetz, SpuRt 2001, 92 - 95

ders., Zur Strafbarkeit von Doping im Sport, JA 2003, 76 - 83

ders., Zum Rechtsgut einer Strafnorm gegen Selbst-Doping, SpuRt 2007, 153 - 155

Hoffmann-Becking, Michael/ Rawert, Peter, Beck'sches Formularbuch. Bürgerliches, Handels- und Wirtschaftsrecht, 9. Aufl. München 2006

Holzer, Wolfgang/ Fritzweiler, Jochen, Auswirkungen von Dopingverstössen auf Arbeits-, Lizenz- und Sponsorenverträge, in: Fritzweiler (Hrsg.), Doping. Sanktionen Beweise Ansprüche, Bern 2000, S. $57-78$

Humberg, Andreas, Die Möglichkeit zur außerordentlichen Kündigung eines Sponsoringvertrages, JR 2005, 271 - 274

Leipold, Klaus, Strafrechtliche Aspekte des Doping, NJW-Spezial 2006, $423-424$

Horst, Johannes/ Jacobs, Constanze, Arbeits- und verbandsrechtliche Konsequenzen des Dopings, RdA 2003, 215 - 222

Joecks, Wolfgang/ Miebach, Klaus, Münchener Kommentar zum Strafgesetzbuch

- $\quad$ Band 5, Nebenstrafrecht I, München 2007

zitiert: MünchKommStGB-Bearbeiter

Kargl, Walter, Begründungsprobleme des Dopingstrafrechts, NStZ 2007, 489 - 496

Köbler, Gerhard, Juristisches Wörterbuch. Für Studium und Ausbildung, 13. Aufl. München 2005

Kolvenbach, Dirk W., Zivilrechtliche Ausgestaltung von Sponsoringverträgen - «Was man unbedingt beachten sollte»-, AnwBl 1998, 289

$-304$ 
Kratz, Alexandra/ Quantius, Markus, Zur außerordentlichen Kündigung von Sportsponsoringverträgen in Dopingfällen, in: Bepler (Hrsg.), Sportler, Arbeit und Statuten: Herbert Fenn zum 65. Geburtstag, Berlin 2000, S. 177 - 197

Kübl, Kristian, Zur strafrechtlichen Relevanz sportethischer Beurteilung des Dopings, in: Vieweg (Hrsg.), Doping. Realität und Recht, Berlin 1998, S. 77 - 87

Lange, Hermann/ Schiemann, Gottfried, Schadensersatz, 3. Aufl. Tübingen 2003

Lieb, Manfred, Das Leitbild des Finanzierungsleasing - Leasing im Spannungsfeld von Vertragsfreiheit und Inhaltskontrolle, DB 1988, $946-954$

Menzel, Tobias, Dopingsperren durch Sportverbände als Missbrauch einer marktbeherrschenden Stellung, WRP 2002, 810 - 816

Mertens, Karsten, Jan Ullrich und die Unschuldsvermutung, SpuRt 2006, $177-180$

Nesemann, Tim, Vertragsstrafen in Sponsoringverträgen im Zusammenhang mit Doping, NJW 2007, 2083 - 2086

Netzle, Stephan, Wie hält es das Internationale Sportschiedsgericht mit dem Doping?, in: Vieweg (Hrsg.), Doping. Realität und Recht, Berlin 2000, S. 197 - 218

Oechsler, Jürgen, Gerechtigkeit im modernen Austauschvertrag. Die theoretischen Grundlagen der Vertragsgerechtigkeit und ihr praktischer Einfluß auf Auslegung, Ergänzung und Inhaltskontrolle des Vertrages, Tübingen 1997

Otten, Ralf Gerhard, Sponsoring: Erscheinungsformen, Rechtsgrundlagen und Bedeutung für die Finanzierung des öffentlich-rechtlichen Rundfunks, München 2000

Otto, Harro, Zur Strafbarkeit des Doping - Sportler als Täter und Opfer, SpuRt 1994, 10 - 16

Partikel, Andrea, Formularbuch für Sportverträge. Vereine und Gesellschaften, Dienst- und Arbeitsverhältnisse, Sportanlagen, Sportdienstleistungen, Veranstaltungen, Werbung und Sponsoring, 2. Aufl. München 2006

Petri, Grischka, Die Sanktionsregeln des World-Anti-Doping-Codes, SpuRt 2003, $230-234$

ders., Das Instrument der Schutzsperre und seine Legitimität, SpuRt 2006, $105-108$ 
Prütting, Hanns/ Wegen, Gerhard/ Weinreich, Gerd (Hrsg.), BGB

Kommentar, 3. Aufl. Köln 2008

zitiert: Prütting/ Wegen/ Weinreich-Bearbeiter

Rebmann, Kurt/ Säcker, Franz Jürgen/ Rixecker, Roland (Hrsg.), Mün-

chener Kommentar zum Bürgerlichen Gesetzbuch

- $\quad$ Band 2a, SS 241 - 432, 5. Aufl. München 2007

- $\quad$ Band 4, Schuldrecht Besonderer Teil II (\$S 611 - 704),

4. Aufl. München 2005

zitiert: MünchKommBGB-Bearbeiter

Röhrborn, Stefan, Der Sponsoringvertrag als Innengesellschaft.

Abschluß, Vollzug und Leistungsstörungen, Frankfurt a.M./ Ber-

lin/ Bern/ New York/ Paris/ Wien 1997

Schaub, Renate, Sponsoring und andere Verträge zur Förderung überindividueller Zwecke, Tübingen 2008

Schild, Wolfgang, Doping in strafrechtlicher Sicht, in: Schild (Hrsg.),

Rechtliche Fragen des Dopings, Heidelberg 1986, 13 - 34

Schütze, Rolf A./ Weipert, Lutz (Hrsg.), Münchener Vertragshandbuch, Band 3 Wirtschaftsrecht II, 5. Aufl. München 2004

zitiert: Münchener Vertragshandbuch-Bearbeiter

Schwab, Dieter, Zivilrechtliche Haftung beim Doping, in: Schild

(Hrsg.), Rechtliche Fragen des Dopings, Heidelberg 1986, S. 35 50

Standinger, Julius von, Kommentar zum Bürgerlichen Gesetzbuch,

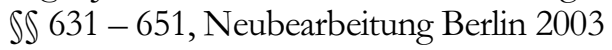

Steinigen, Jens, Zivilrechtliche Aspekte des Dopings aus der Sicht des

Spitzensportlers, Berlin 2003

Stoffels, Markus, Gesetzlich nicht geregelte Schuldverträge. Rechtsfin-

dung und Inhaltskontrolle, Tübingen 2001

Tilch, Horst/ Arloth, Frank (Hrsg.), Deutsches Rechtslexikon, 3. Aufl.

München 2001

Turner, George, Doping und Zivilrecht, NJW 1992, 720 - 723

ders., Doping und Zivilrecht, in: Festgabe Zivilrechtslehrer 1934/35, Berlin 1999, 669 - 682

Vieweg, Klaus, Doping und Verbandsrecht, NJW 1991, 1511 - 1516

ders., Dopingvermeidung und Verbandsrecht - Regelkreismodell, Ergebnisse und Analyse einer explorativen Erhebung, in: Vieweg (Hrsg.), Doping. Realität und Recht, Berlin 2000, S. 113 - 133 
Walker, Wolf-Dietrich, Die Vertragsstrafe im Arbeitsvertrag des Sportlers am Beispiel des Lizenzfußballspielers, in: Crezelius (Hrsg.), Festschrift für Volker Röhricht: Gesellschaftsrecht, Rechnungslegung, Sportrecht, Köln 2005, S. 1277 - 1296

ders., Beweisrechtliche und arbeitsrechtliche Probleme des Dopings, in:

Vieweg (Hrsg.), Doping. Realität und Recht, Berlin 1998, S. 135 175

Weber, Klaus (Hrsg.), Creifelds, Rechtswörterbuch, 19. Aufl. München 2007

Weiand, Neil George, Kultur- und Sportsponsoring im deutschen Recht, unter besonderer Berücksichtigung urheber-, medien- und wettbewerbsrechtlicher Aspekte, Berlin 1993

ders., Rechtliche Aspekte des Sponsoring, NJW 1994, 227 - 233

Weiand, Neil George/ Poser, Ulrich, Sponsoringvertrag, 3. Aufl. München 2005 


\section{De}

ie zunehmende Aufdeckung von Dopingfällen im Spitzensport hat zu einer Verstärkung der Diskussion über juristische Aspekte des Dopings geführt. Bisher standen dabei strafrechtliche sowie vereins- bzw. verbandsrechtliche Fragen im Mittelpunkt. In dieser rechtsdogmatischen Abhandlung soll eine Problematik in den Vordergrund gestellt werden, deren praktische Relevanz die zahlreichen Berichte über Rückzüge von Sponsoren der Tour de France verdeutlichen: die Frage, ob und wie Sponsoringverträge in Fällen von Verfehlungen des Gesponserten beendet werden können. Zum einen wird untersucht, wie entsprechende Rücktrittsklauseln beschaffen sein sollten. Zum anderen wird geprüft, unter welchen Voraussetzungen auch ohne ausdrückliche Regelungen für Dopingfälle eine Vertragsbeendigung nach allgemeinen schuldrechtlichen Grundsätzen in Betracht kommt. Ebenso wird auf die Rechtsfolgen einer Vertragsbeendigung eingegangen, insbesondere auf ihr Verhältnis zu anderen vertragsrechtlichen Folgen des Dopings, etwa Schadensersatzansprüchen oder Vertragsstrafen.

ISBN 978-3-940344-54-0

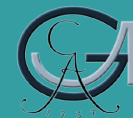

